\title{
Review Article \\ Sleep Spindle Characteristics in Children with Neurodevelopmental Disorders and Their Relation to Cognition
}

\author{
Reut Gruber' ${ }^{1}$ and Merrill S. Wise ${ }^{2}$ \\ ${ }^{1}$ Department of Psychiatry, McGill University, 6875 LaSalle Boulevard, Montreal, QC, Canada H4H 1R3 \\ ${ }^{2}$ Methodist Healthcare Sleep Disorders Center, 5050 Poplar Avenue, Memphis, TN 38157, USA \\ Correspondence should be addressed to Reut Gruber; reut.gruber@douglas.mcgill.ca
}

Received 30 December 2015; Revised 11 March 2016; Accepted 26 April 2016

Academic Editor: Julie Seibt

Copyright (c) 2016 R. Gruber and M. S. Wise. This is an open access article distributed under the Creative Commons Attribution License, which permits unrestricted use, distribution, and reproduction in any medium, provided the original work is properly cited.

\begin{abstract}
Empirical evidence indicates that sleep spindles facilitate neuroplasticity and "off-line" processing during sleep, which supports learning, memory consolidation, and intellectual performance. Children with neurodevelopmental disorders (NDDs) exhibit characteristics that may increase both the risk for and vulnerability to abnormal spindle generation. Despite the high prevalence of sleep problems and cognitive deficits in children with NDD, only a few studies have examined the putative association between spindle characteristics and cognitive function. This paper reviews the literature regarding sleep spindle characteristics in children with NDD and their relation to cognition in light of what is known in typically developing children and based on the available evidence regarding children with NDD. We integrate available data, identify gaps in understanding, and recommend future research directions. Collectively, studies are limited by small sample sizes, heterogeneous populations with multiple comorbidities, and nonstandardized methods for collecting and analyzing findings. These limitations notwithstanding, the evidence suggests that future studies should examine associations between sleep spindle characteristics and cognitive function in children with and without NDD, and preliminary findings raise the intriguing question of whether enhancement or manipulation of sleep spindles could improve sleep-dependent memory and other aspects of cognitive function in this population.
\end{abstract}

\section{Introduction}

Neurodevelopmental disorders (NDDs) are a heterogeneous group of conditions in which the development of the central nervous system is disrupted. Manifestations can include impairments in motor function, learning, cognition and/or communication, or neuropsychiatric problems. These issues appear early in development, persist throughout life, and produce notable impairments in social, communicative, cognitive, and behavioral functioning [1] that can vary from very specific limitations to global impairment in intelligence and social skills. This group of disorders includes intellectual disability (formerly referred to as mental retardation), communication disorders, autism spectrum disorder (ASD), Attention Deficit Hyperactivity Disorder (ADHD), specific learning disorders, and neurodevelopmental motor disorders including cerebral palsy (CP).

Sleep is a vital process for brain restoration and it is critical for maintaining cognitive function. Strong empirical evidence indicates that sleep spindles facilitate the plasticity which supports learning, memory consolidation, declarative learning, motor skills, and overall intellectual performance. The cognitive functions that are related to sleep spindles are also key domains of dysfunction in children with NDD [2].

Clinically significant sleep problems are prevalent in children and adolescents with NDD [3, 4]. Among children with neurocognitive difficulties, the impact of disrupted sleep spindle generation may be amplified. Hence, the characteristics of NDD may increase both the risk for and vulnerability to abnormal sleep spindle generation. The goal of the present review is to review and integrate existing evidence regarding sleep spindle characteristics in children with NDD and, when evidence is available, to examine the associations between these characteristics and cognitive function.

A better understanding of relationships between sleep and NDD is expected to provide insight into the pathophysiology and possibly the treatment of such disorders and 
improve our understanding of the association between sleep spindles and cognition in children. It is likely that sleep spindle characteristics represent a marker of brain development and function, as well as a window into underlying brain mechanisms that support cognition.

We will first briefly describe sleep spindle development and its relationship with cognitive development and function in typically developing children. We will then review the available evidence regarding sleep spindle characteristics in children with a variety of NDDs and their relation to cognition. Finally, we will discuss the findings, identify gaps in understanding, and recommend future research directions in this emerging area of investigation.

\section{Sleep Spindles in Typically Developing Children}

Sleep spindles represent an oscillating electrical potential in the brain; they have a characteristic frequency of $11-16 \mathrm{~Hz}$ (usually $12-14 \mathrm{~Hz}$ in healthy adults) and last from one to several seconds in duration [5]. On scalp electroencephalography (EEG), spindles are seen as sinusoidal waves that often have a fusiform or "crescendo-decrescendo" morphology [6].

Sleep spindles are characterized by their symmetry; synchrony between hemispheres; amplitude, which is the peak-to-peak difference in spindle size, reflecting voltage; frequency, which is the number of waveforms per second; density, which is the number of spindle bursts/min of NREM sleep; and the duration of spindle bursts. In infants, sleep spindles last several seconds in duration, are expressed maximally in the frontocentral location, are in the high alpha or low beta frequency range, and are not synchronous. The lack of synchrony is likely due to lack of myelination in the neonatal brain. By 2 years of age, it is considered abnormal if most spindles are still asynchronous [7]. In older children and adults, sleep spindles are expressed diffusely across the head but maximally over the central regions and in a bilaterally synchronous and symmetric fashion [7]. Moreover, sleep spindles can be divided into two distinct types based on their frequency and field of expression. Slower spindles (9 to $<13 \mathrm{~Hz}$ ) occur maximally over the frontal regions, whereas faster spindles $(>13-16 \mathrm{~Hz})$ dominate $[8,9]$ over the central and parietal head regions and typically precede slow spindles by hundreds of milliseconds [9-11]. The slow spindles display a typical waxing-and-waning pattern, whereas fast spindles are mainly waning [12]. This difference begins to develop at around 2 years of age [13]. The two populations of sleep spindles are thought to arise from and represent different generators within the thalamus, with some level of cortical involvement. They also demonstrate different maturational patterns, suggesting that their development is associated with changes in thalamocortical structures and maturation of the physiological systems that produce spindles $[6,14$, 15]. Shinomiya et al. suggested that separate investigation of the two types of spindles may be important in evaluating developmental processes in the central nervous systems of children and adolescents, and that frontal spindle activity could represent a good indicator of biological maturation [15].
2.1. Generation of Sleep Spindles. Sleep spindles are a prototypical thalamocortical rhythm generated and "paced" in the thalamus via a network of synaptic interactions involving inhibitory (GABAergic) neurons in the reticular thalamic nuclei, thalamocortical cells, and cortical pyramidal neurons [16]. In animal models, the spindle rhythm is abolished by destruction of the thalamus, whereas it persists after decortication when the thalamus is preserved [14, 17]. Spindle frequency is determined in large part by an interplay between mutually interconnected GABAergic inhibitory neurons of the reticular nucleus of the thalamus and the thalamocortical neurons, with influence from inputs of the cortex and brainstem [14]. Reticular thalamic neurons impose hyperpolarization on thalamocortical neurons, thereby activating a nonspecific cation current that depolarizes the thalamocortical neurons and activates low-threshold calcium ion currents and bursting. The latter process provides feedback excitation to reticular thalamic neurons, thus closing the loop. Each thalamocortical burst also imposes an excitatory postsynaptic potential (EPSP) on pyramidal neurons, providing the basis for the spindle waveforms observed on scalp EEG.

2.2. Development of Sleep Spindles. In typically developing children, very early ("rudimentary") spindles may be observed as early as term to 2 weeks postterm $[15,18-$ 21] and it was proposed that they could appear earlier in premature infants [22]. Spindles become more easily identified between 3 and 9 weeks postterm, when they often occur in relatively long trains lasting up to 10 seconds during quiet sleep $[21,23,24]$. In the first 6 months, spindles may occur unilaterally, often alternating between hemispheres. Asymmetry is common as well. Spindles become increasingly more synchronous between hemispheres during the first year of life, reflecting maturation of interhemispheric connections [25]. By 12 to 18 months, most sleep spindles are expressed in a bilaterally synchronous and symmetric fashion, with maximal expression over the central regions.

Maturation alters sleep spindle activity in terms of the spindle frequency, amplitude, duration, and density $[15,18-$ $20,26,27]$. Changes in the development of spindle duration and density are thought to follow a U-shaped distribution, whereas that of the interspindle interval shows an inverted U-shape. Three distinct phases of sleep spindle development have been proposed: (1) infants up to 9-10 months old exhibit long spindles (around $1.5 \mathrm{~s}$ ) having a relatively low density $(<3 / \mathrm{min})$ and a relatively short interspindle interval (around $20 \mathrm{~s}$ ); (2) children from 10 months up to 3 years exhibit a decrease in the spindle length (to around $0.8 \mathrm{~s}$ ) and an increase in the interspindle interval (up to $111 \mathrm{~s}$ ), yielding an even lower spindle density (0.3-1.2/min); and (3) over three years of age, children show short interspindle intervals (5$10 \mathrm{~s})$ and long spindle durations (0.9-1.5 s) of high density (4-10/min). These changes presumably reflect developmental changes in thalamocortical structures and maturation of the physiological system that produces spindles [26, 27]. Sleep spindle peak frequencies increase from childhood to adolescence $[15,28,29]$, when global maturational changes in sigma power predominate in the slow sigma frequency band [29]. The topographic representation of sigma power 
provides insight into these age-related changes [30] by showing that the fast sigma power increases over the centroparietal areas, while the slow sigma power decreases over frontal areas across childhood and adolescence [15, 30]. Given the association between neural maturation and changes in sleep spindle activity, it has been proposed that sleep spindles could be used as a potential index of neural maturation [27].

\subsection{Sleep Spindles and Cognitive Development in Typically} Developing Children. Intellectual ability is closely related to cortical development in children and adolescents. Intelligence is associated with the trajectory of cortical development, primarily that of the frontal regions which are implicated in the maturation of intelligence [31, 32]. Synaptic density increases until around puberty (11 years for girls and 12 years for boys), whereupon synaptic pruning begins [33]. In fact, vigorous cortical thinning by early adolescence has a positive association with IQ [34].

A similar pattern is seen in the developmental changes of sleep spindles. Initially, during development, the increased neuronal connectivity results in higher sleep EEG amplitudes because the size of these waves reflects the number of synaptic connections [31,35-37]. Thereafter, pruning results in smaller neuronal populations that oscillate in unison, with corresponding decreases in EEG power. These changes in the brain appear to parallel the rapid development of cognitive abilities at similar ages [31,33, 38-43]. Synaptic pruning and increasing myelination during adolescence result in faster and more efficient information processing, which is manifested by an increased ability to perform complex cognitive operations, increased speed and efficiency in completing simple information-processing tasks, and improved performance on intelligence tests across adolescence [37].

Slow spindles have been correlated with visual perceptual learning [44], while fast spindles have been correlated with more complex abilities and processes, such as fluid intelligence [45], learning ability [46] and word-location associations [47]. It has been proposed that they could be used as a neurobiological indicator for the level of cognitive development.

\subsection{Sleep Spindles and Cognitive Function in Typically Devel-} oping Children. The processes underlying sleep spindles have been hypothesized to benefit cognitive functions and "offline" information processing in several ways. First, it is assumed that sleep spindles serve as a "gating mechanism" to protect sleep from being interrupted by external stimuli, such as noise [48], thereby allowing optimal time for off-line information processing. Historically, thalamocortical (TC) cells were thought to gate sensory transmission by switching from tonic to burst discharge mode [16]. The bursting pattern occurs during NREM sleep in the form of sleep spindles, while the tonic pattern of activity occurs in wakefulness. The tonic activity pattern was thought to relay sensory information to the cortex via the thalamus from a variety of afferent inputs, while the bursting or spindle pattern was thought to serve a gating role. More recently, researchers have proposed that both modes can relay stimuli to the cortex. However, while tonic spikes reliably transmit information, the stereotyped discharge profile of bursts leads to nonlinear distortion of sensory inputs [49]. Burst firing of TC cells during spindles would thus filter external stimuli. It has been proposed that one way in which sleep spindles could support cognitive function is by blocking interference (i.e., performing a gating function) to allow uninterrupted off-line processing and consolidation of information.

Sleep spindles appear to actively enhance information processing via their role in memory consolidation. Behavioral studies in animal models and humans have shown that learning improves more during and following a period of sleep than during an equivalent amount of waking time [50-52]. Neuroimaging studies have shown that the patterns of brain activity elicited during initial learning are replayed during subsequent sleep, demonstrating the presence of dynamic offline information processing [53]. Underlying this enhancement or stabilization of memory across a sleep period is the concept of sleep-dependent memory consolidation, whereby memory traces become more stable and resistant to interference over a period of time. The consolidation of declarative memory results from a dialog between reactivated hippocampal memory traces and the neocortical networks that retain long-term memory representations [54]. In adults, sleep-dependent consolidation of declarative memory has been correlated with a range of neurophysiological measures, including the amounts of slow wave activity $(1-4 \mathrm{~Hz})$, slow oscillations $(0.5-1 \mathrm{~Hz})$, and sleep spindles. This indicates that sleep plays an active role in the hippocampal-neocortical dia$\log$ [54]. Recent research conducted in children of different ages has shown that their sleep-dependent consolidation of declarative memory is comparable to that of adults, whereas that of procedural skills is not $[55,56]$.

One explanation for how sleep spindles contribute to new learning is related to the hypothesis that sleep spindles facilitate the priming of synapses for plastic changes, [57] thereby enabling the off-line information processing that is essential for learning to be completed [58]. Data from simulations of sleep spindle activity have indicated that repetitive thalamic bursts (similar to sleep spindle activity) generate robust entry of $\mathrm{Ca}^{2+}$ into cortical dendrites [59], which produces conditions that favor the priming of synapses for plastic changes (i.e., by activating proplastic signaling molecules such as protein kinase $\mathrm{A}$ and $\mathrm{Ca}^{2+} /$ calmodulin-dependent protein kinase II). The synaptic potentiation induced by spindles is consistent with the hypothesis of "active system consolidation" [60], which proposes that sleep spindles potentiate memory traces by reactivating selected neuronal circuits.

In addition to the contribution of sleep spindles to creating the conditions that allow for optimal sleep (i.e., by gating), brain plasticity, and memory consolidation, the characteristics of sleep spindles have been shown to be stable within subjects across different nights [6], and to correlate with intellectual ability, particularly fluid intelligence. This has led to the view that spindles, at least under non-pathological conditions, constitute to some extent a biophysical measure of intelligence.

In children, mixed results have been reported in regard to the direction and strength of associations between the different characteristics of sleep spindles and performance 
on intelligence tests and memory tasks. Geiger et al. found that peak sleep spindle frequency was negatively correlated with full scale IQ but that relative sigma power correlated positively with full scale IQ and fluid IQ [61]. Gruber et al. showed that lower sleep spindle frequency was associated with better performance on the intelligence perceptual reasoning and working memory WISC-IV scales but that sleep spindle amplitude, duration, and density were not associated with performance on IQ tests [62]. Chatburn et al. observed that the number of fast spindles was positively correlated with narrative memory and negatively correlated with sensorimotor functioning. Mean central frequency of spindles was also negatively correlated with sensorimotor functioning, planning ability, and working memory [63]. Hoedlmoser et al. found that children with higher sleep spindle activity, as measured at frontal, central, parietal, and occipital sites during both baseline and experimental nights, exhibited higher general cognitive abilities (WISC-IV) and declarative learning efficiency (i.e., number of recalled words before and after sleep) [64].

Astill et al. reported that individual differences in the dominant frequency of spindles and slow waves were predictive for performance on finger sequence tapping tasks. Children performed better if they had fewer slow spindles, more fast spindles, and faster slow waves. On the other hand, overnight enhancement of accuracy was most pronounced in children with more slow spindles and slower slow waves, that is, the children with an initial lower performance at baseline. Thus, associations of spindle and slow wave characteristics with initial performance may confound interpretation of their involvement in overnight enhancement. Slower frequencies of characteristic sleep events may be a marker of slower learning and immaturity of networks involved in motor skills [65]. Bódizs et al. found that fluid IQ correlated positively with fast spindle density and amplitude in girls and that these correlations peak in the frontocentral regions. In boys, by contrast, the only positive spindle-index of fluid IQ was found to be the frequency of fast spindles [66]. Doucette et al. observed that children with faster processing speeds exhibited higher slow sigma power over the parietal region [67]. For a detailed description of these findings in typically developing children please see Table 1.

In summary, sleep spindles appear in early infancy, and they change and develop through childhood and adolescence in a progression that parallels the milestones of cortical development. Sleep spindles appear to contribute to off-line information processing by protecting sleep (and thus sleepdependent processes) from being interrupted. They also seem to facilitate the brain plasticity that allows essential learning to occur through processes such as memory consolidation. Finally, certain characteristics of sleep spindles have been correlated with intellectual performance, particularly fluid intelligence.

\section{Sleep Spindles in Children with Neurodevelopmental Disorders}

Despite the high prevalence of sleep problems in children with NDD and the wide range of cognitive deficits in this population, there is only limited information regarding the characteristics and function of sleep spindles in this population. Only a handful of studies have examined sleep in children with NDD, and the existing data are limited in scope and the range of disorders studied. The few existing studies on the putative association between sleep spindles and cognitive function have been conducted in children with intellectual disabilities, ASD, reading disabilities, and ADHD. However, although the body of knowledge is scattered, often based on nonstandardized methods, not always up-to-date, and based mostly on descriptive and correlational studies, it provides important insights into the extent to which sleep spindles are abnormal in these populations compared to typically developing children. Moreover, the existing studies give us an initial view of the nature and extent of the associations between sleep spindles and different intellectual levels in children with neurodevelopmental disorders or (in a few studies) a more direct idea of the associations between sleep spindles and information processing or intellectual performance.

3.1. Sleep Spindles in Children with Intellectual Disability. Intellectual disability is characterized by three findings: an intelligence quotient (IQ) of 75 or below; significant limitations in adaptive behaviors; and onset of disability occurring before age 18. In the past, the term "mental retardation" was used to describe this condition, but this term is no longer used [68]. The common causes of intellectual disability include genetic conditions, brain insults during pregnancy, problems at the time of birth, medical problems that affect brain health, and exposure to environmental toxins (e.g., lead or mercury).

Sleep problems are more severe and more prevalent in children with NDD than in typically developing children. Insomnia in normally developing young children is most often behaviorally based [69], whereas insomnia in children with NDD is more often multifactorial, with neurologic, medical, and psychiatric comorbidities contributing to behavioral issues. Moreover, insomnia in NDD tends to be chronic, often lasting into adolescence or adulthood [70]. Quine showed that sleep problems are persistent in children with developmental disabilities [71], and Wiggs and Stores found that the average duration of sleep problems in such children is 7.13 years (SD 4.04 years) [72].

Alarmingly high prevalence rates for sleep disorders have been cited in children with intellectual disabilities: $86 \%$ in children under 6 years old, $81 \%$ in those aged 6 to 11 years old [71], and 77\% in those aged 12 to 16 years old [73]. Night waking and settling difficulties are particularly common, affecting over half of children with NDD aged up to 16 years. The chronicity of these problems was illustrated by Quine [71], who found that half of children with NDD with settling problems and over two-thirds of those with night waking were still having problems 3 years later.

Sleep disorders are also highly prevalent in children with ASD: recent studies have reported prevalence rates as high as $40-85 \%$ in these children versus $20-40 \%$ in typically developing children [74-76]. The commonly reported sleep disturbances include prolonged sleep-onset latency, restless sleep, frequent nocturnal awakenings, and reduced total sleep 


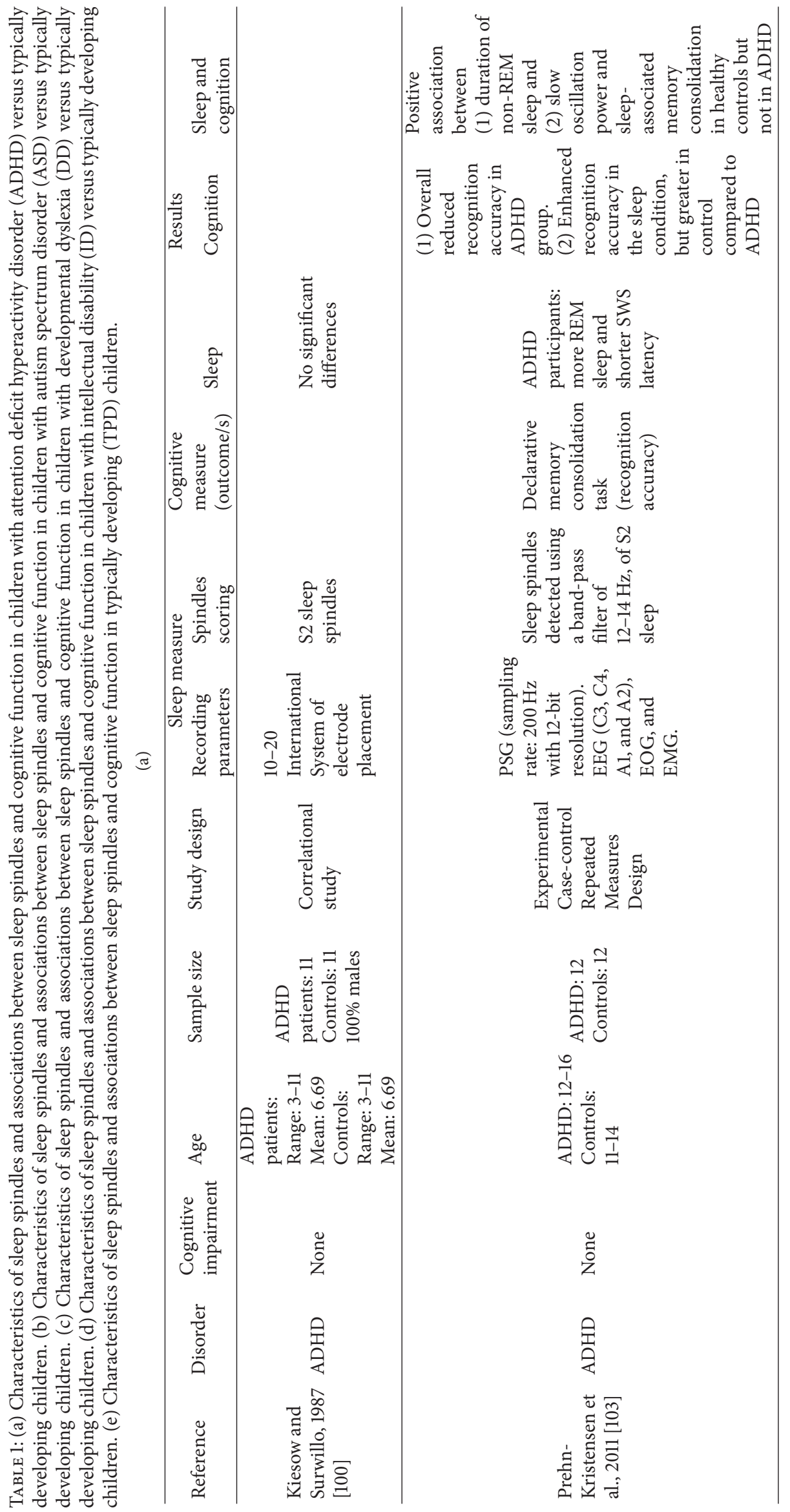




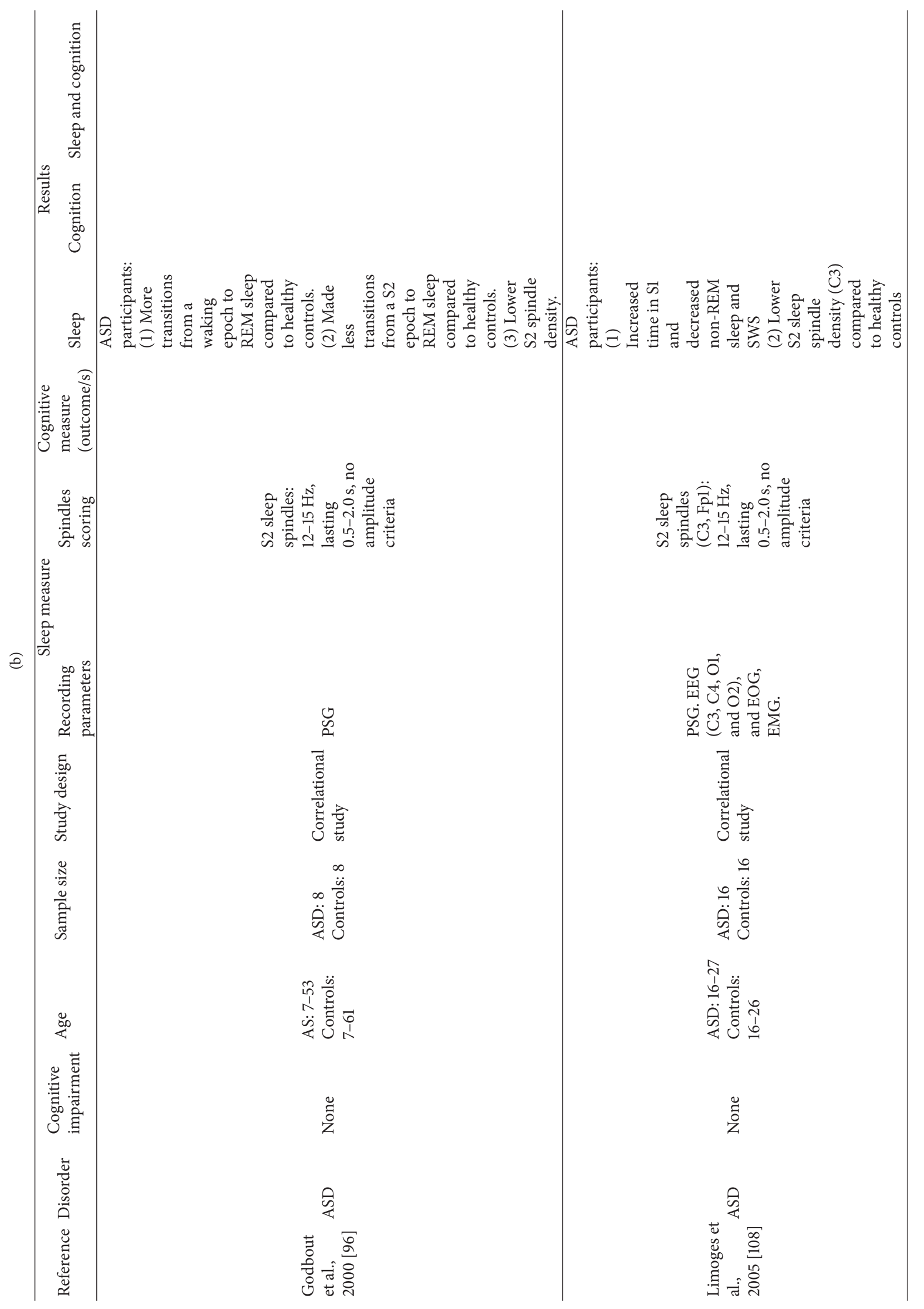




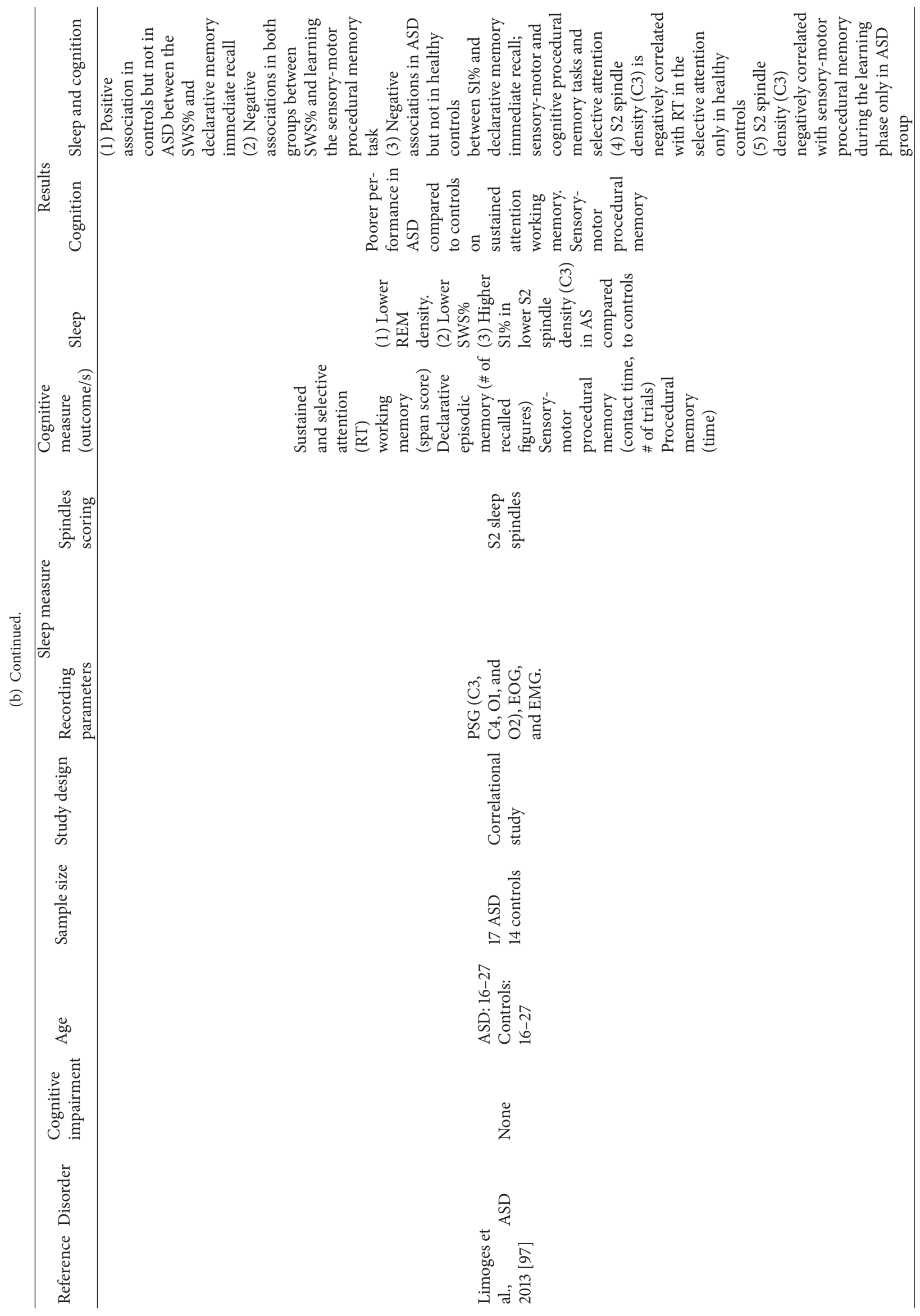




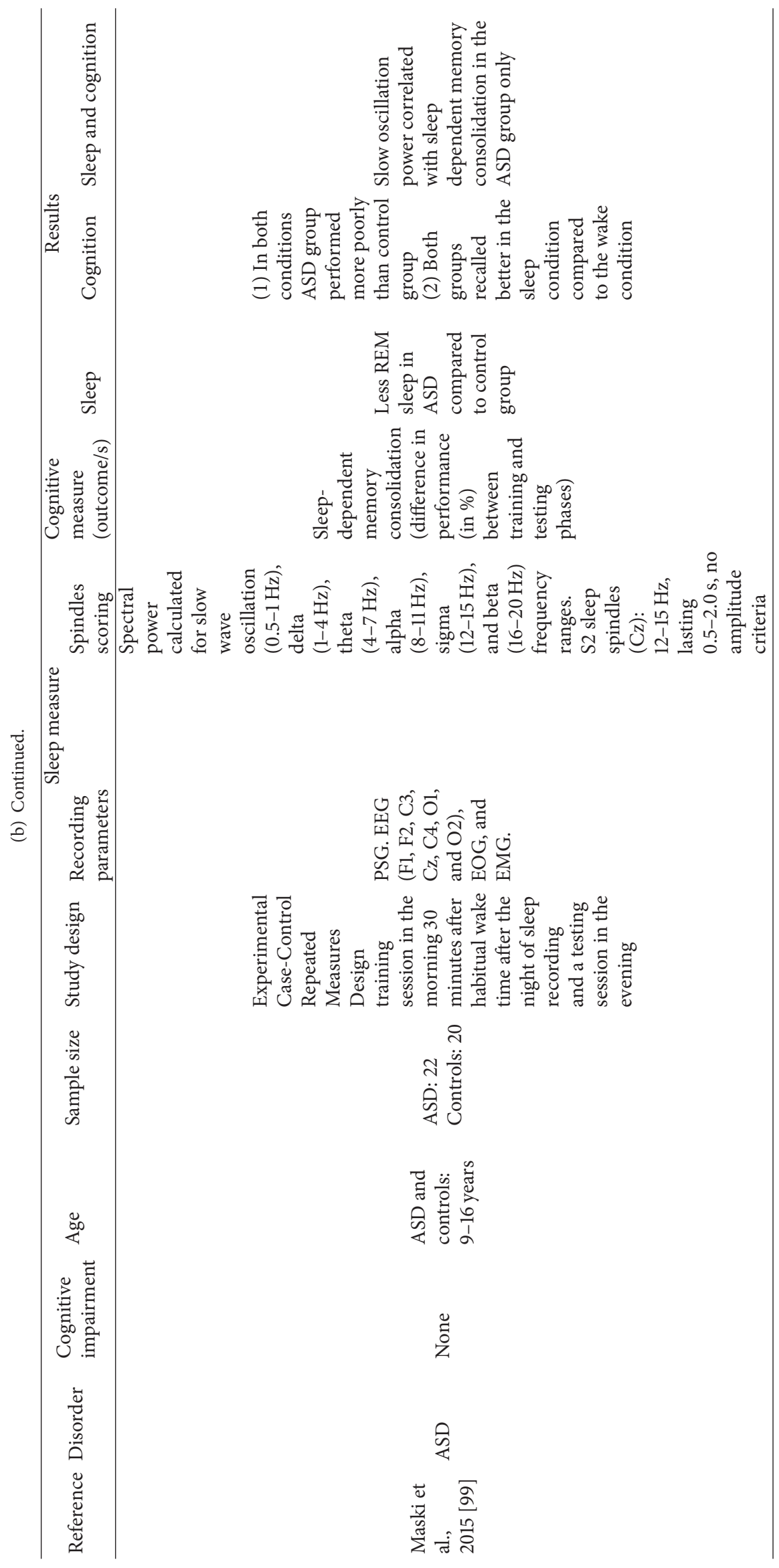




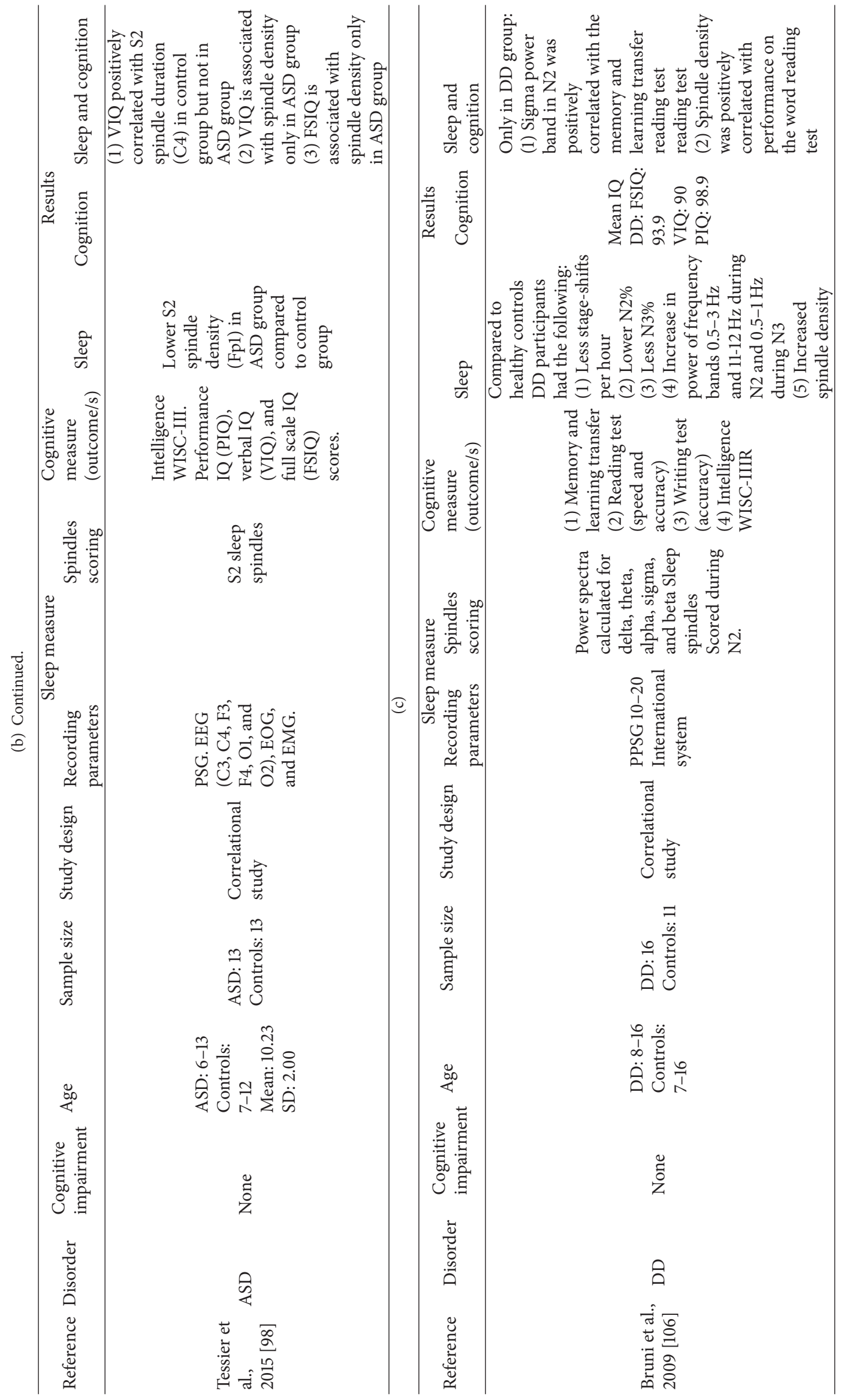




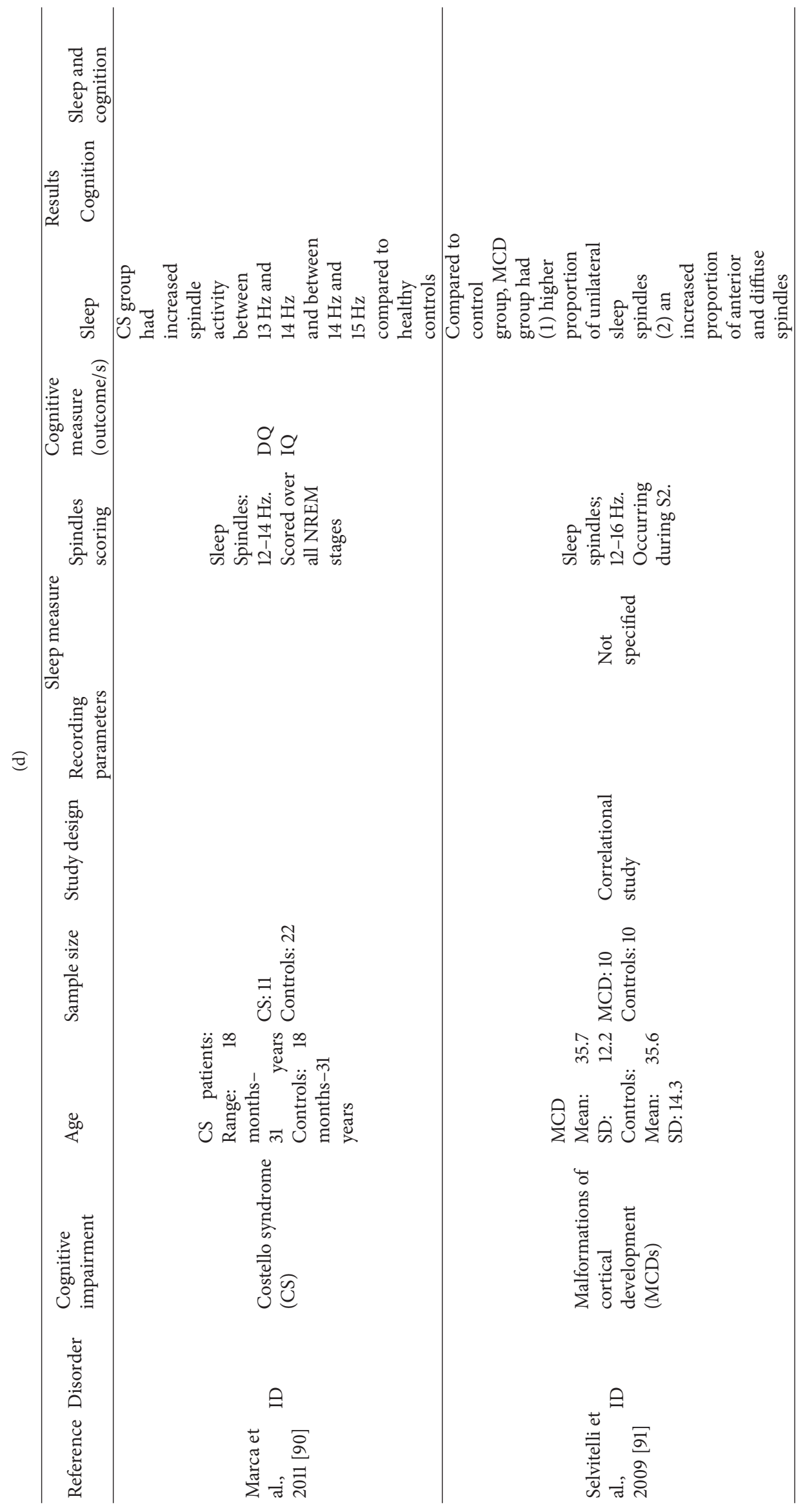




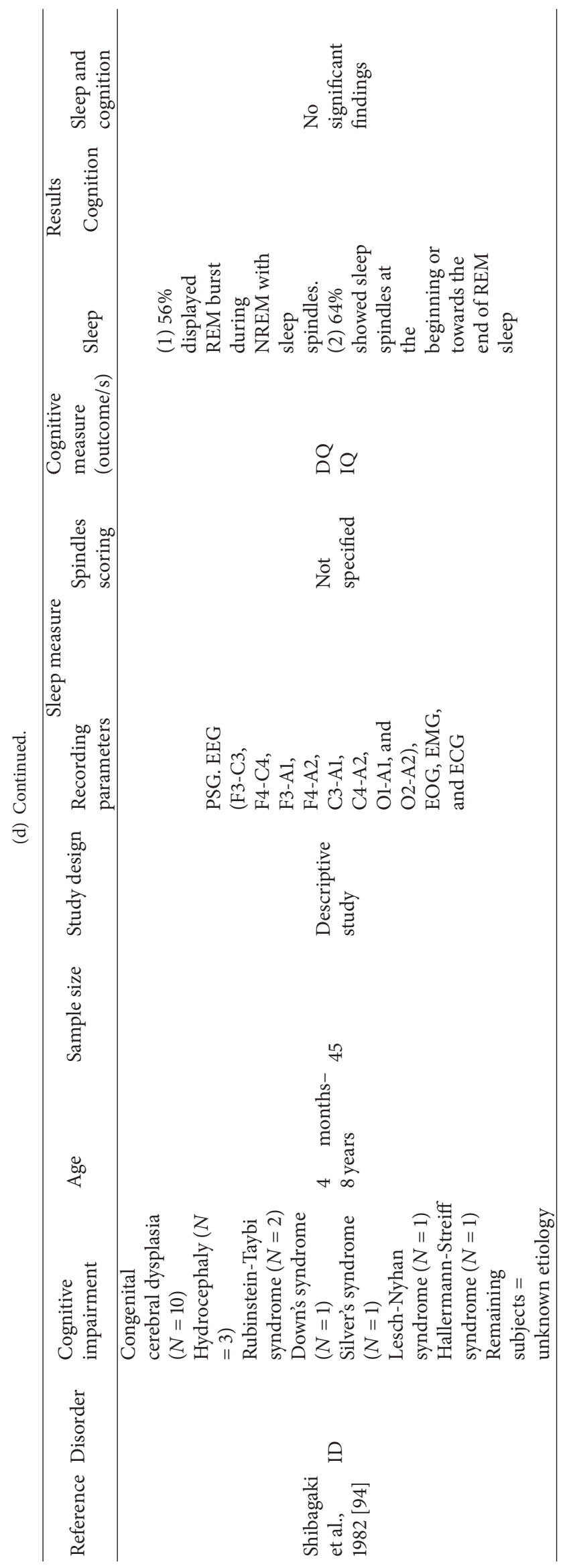




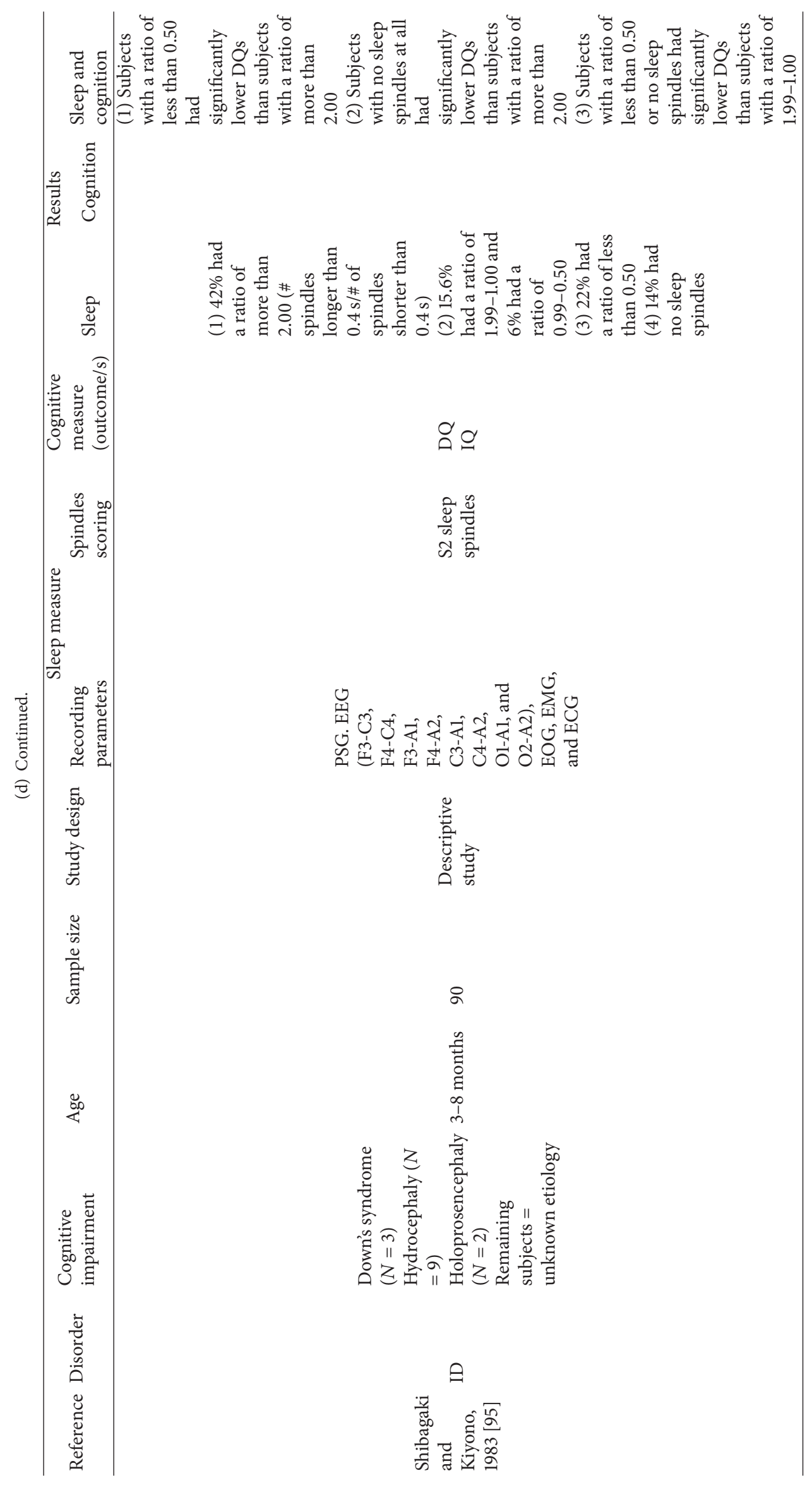




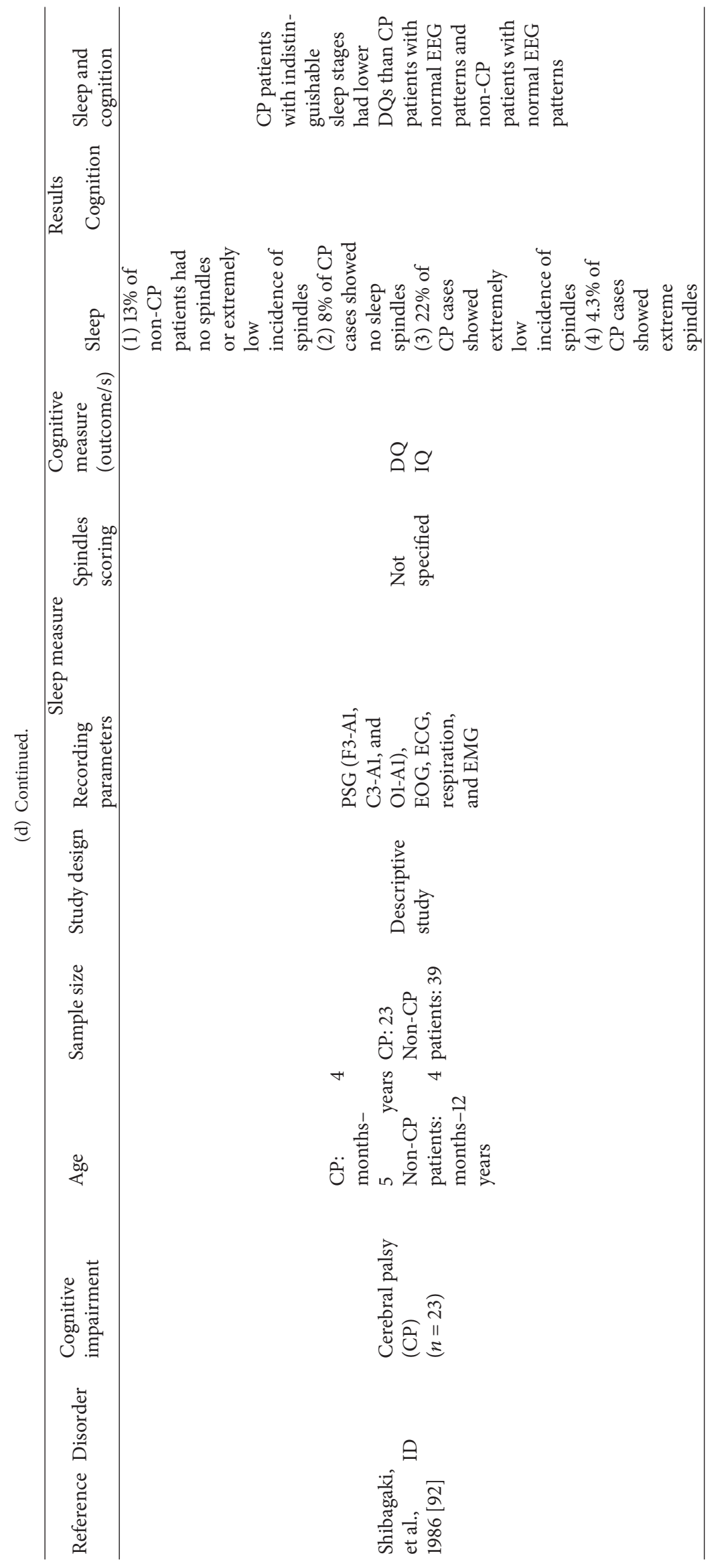




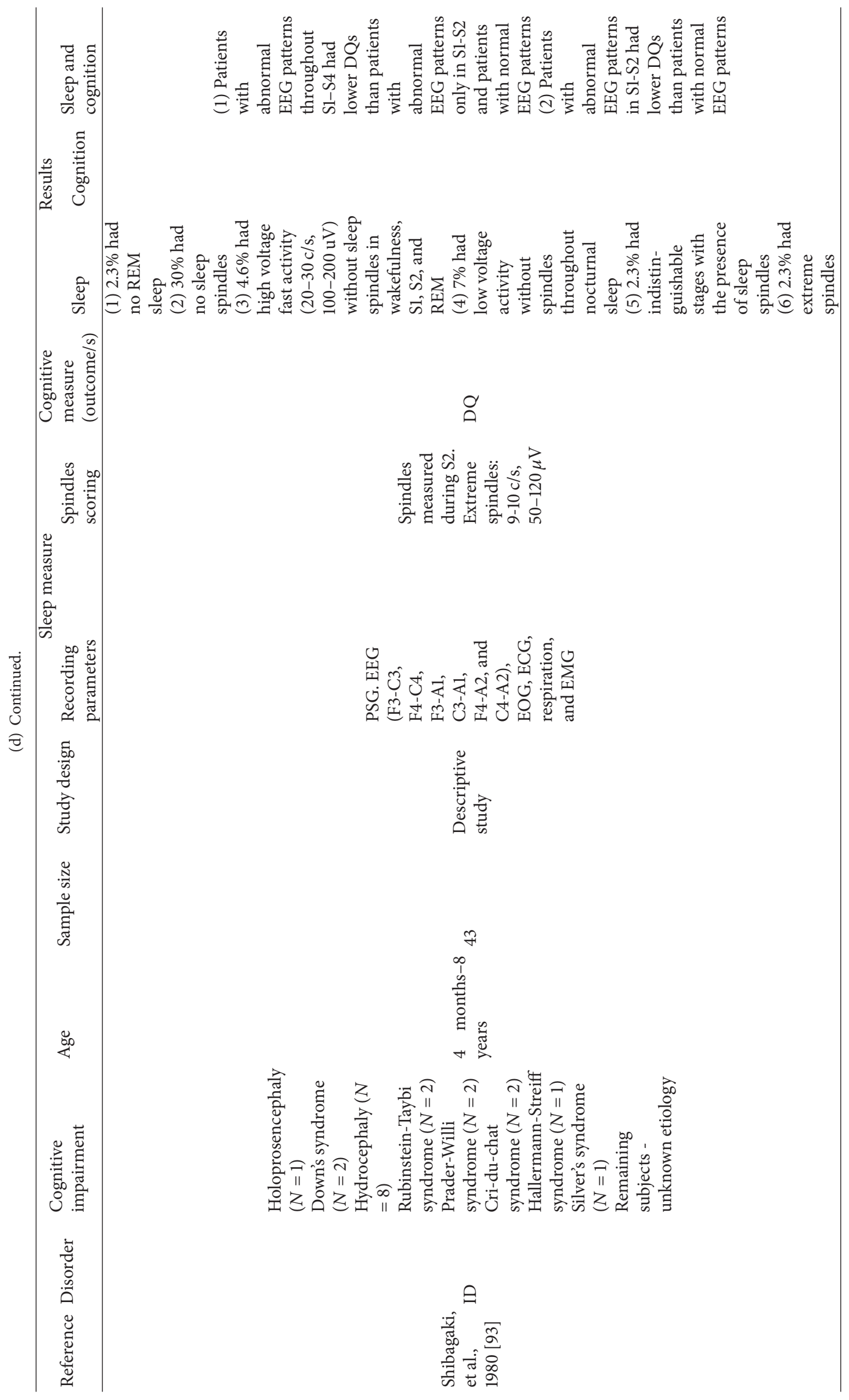




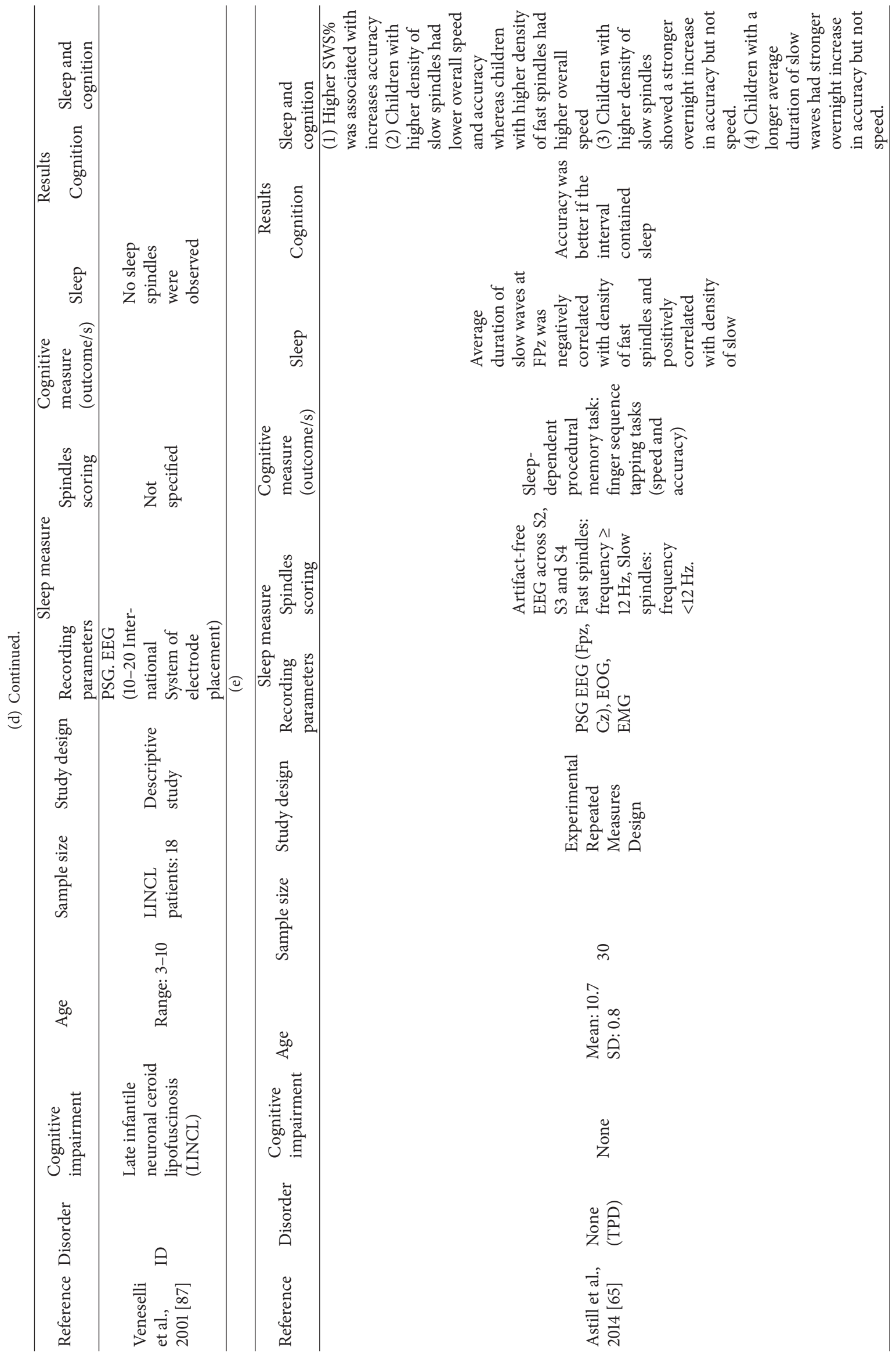




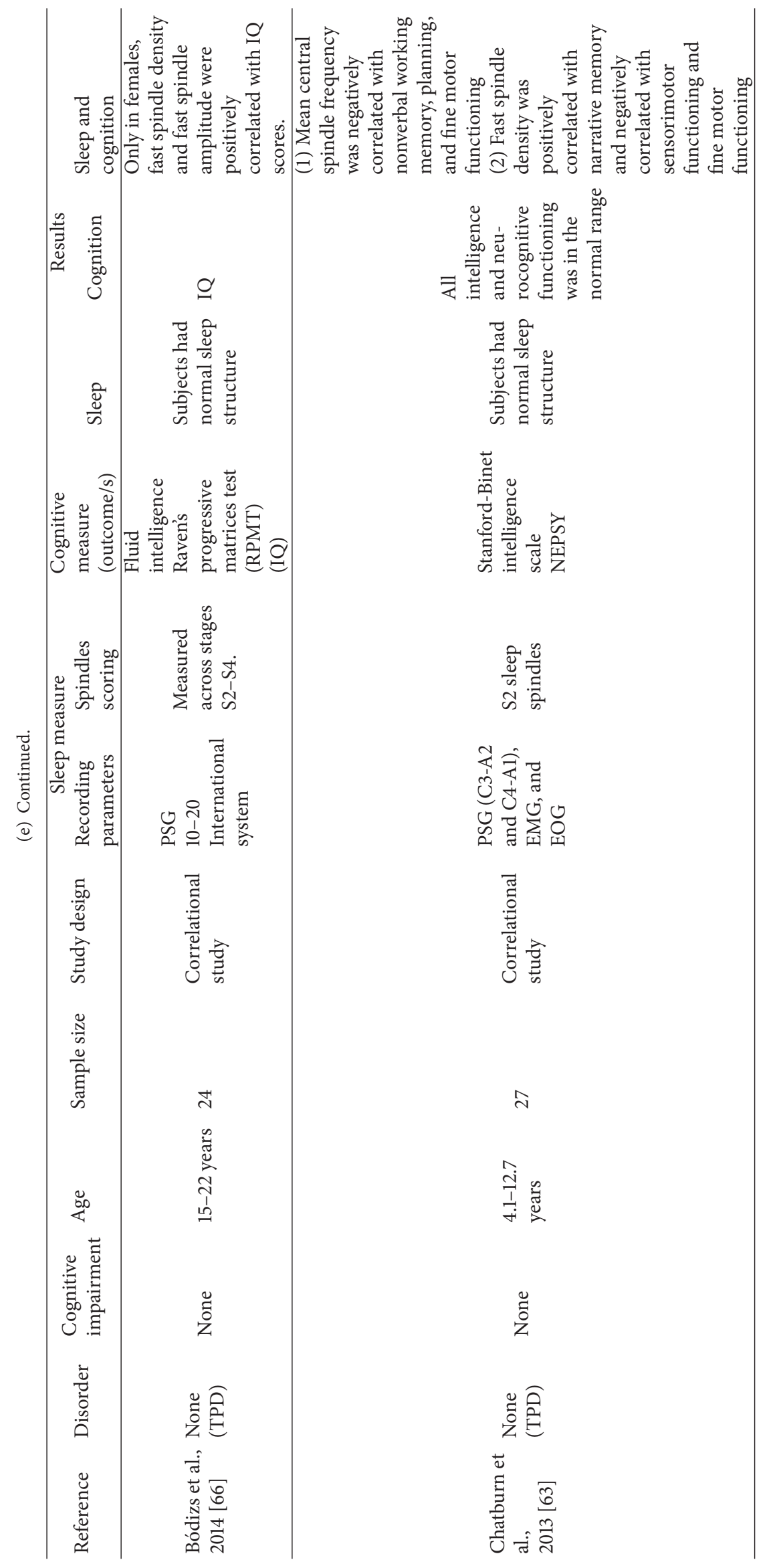




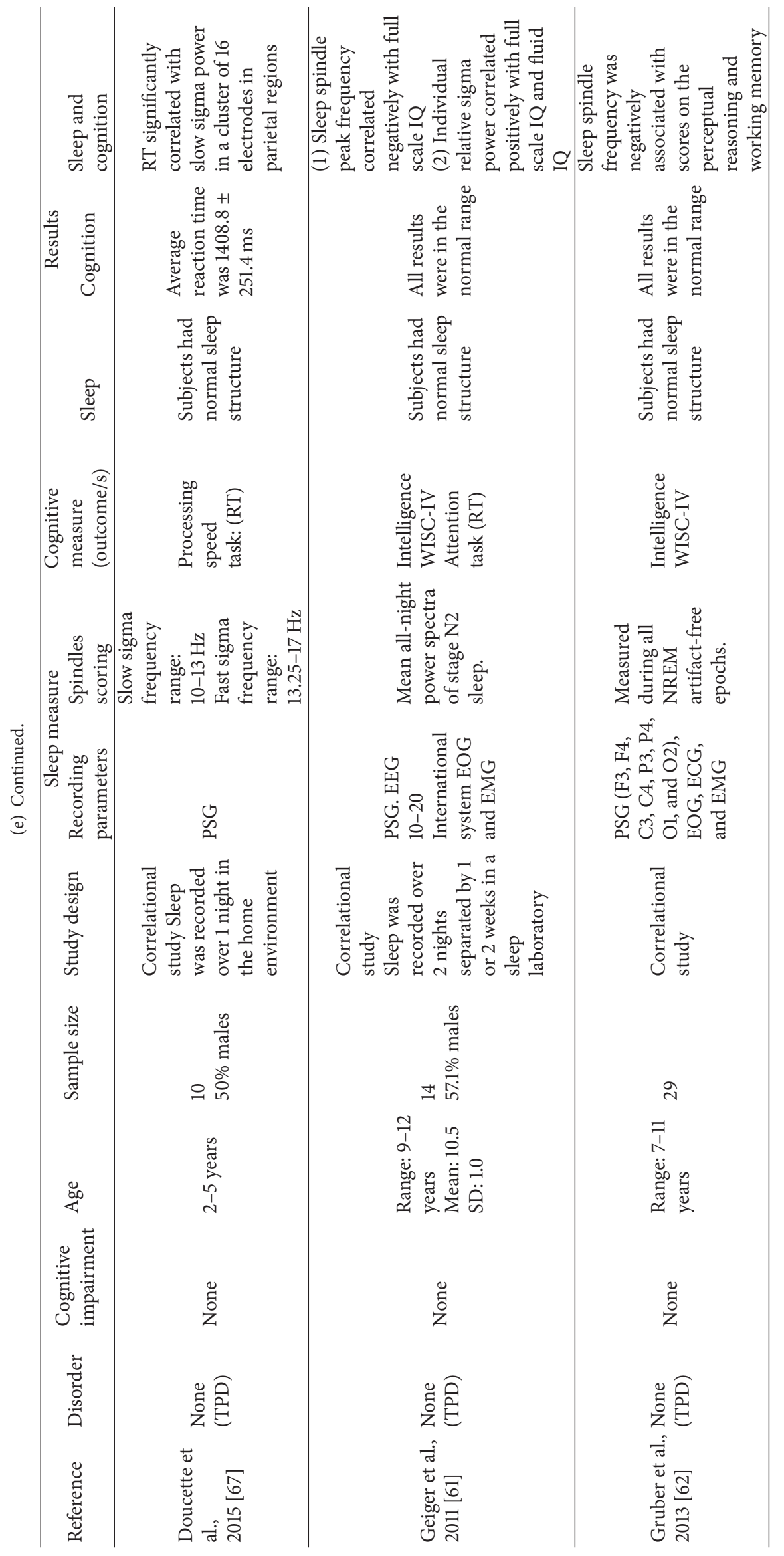




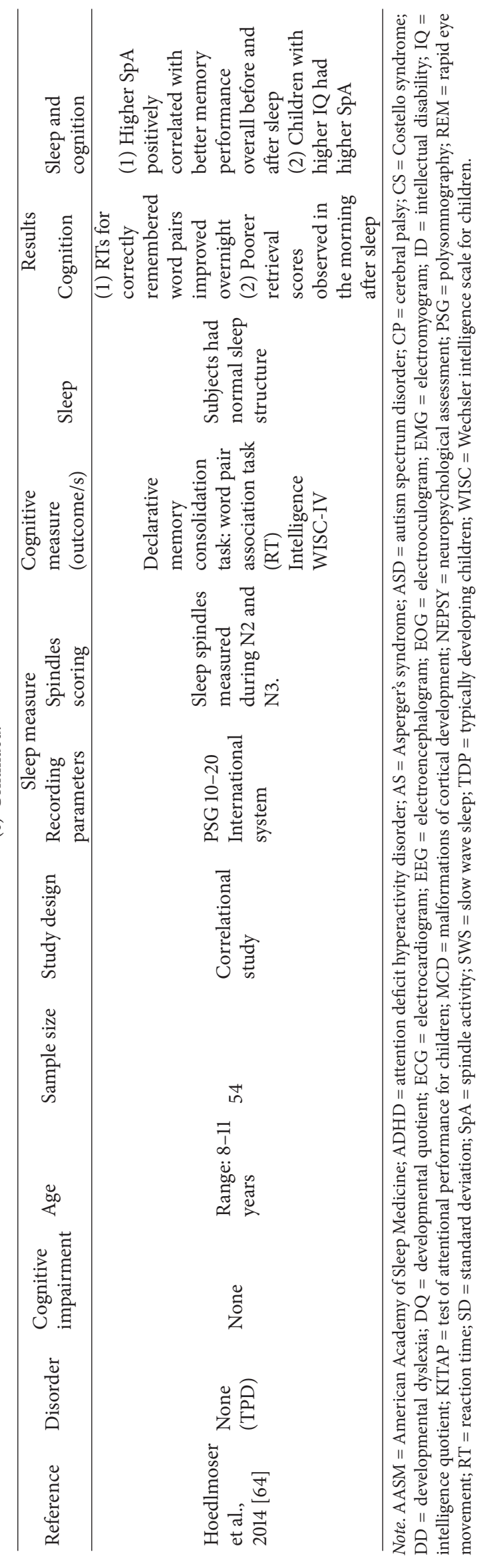


time. Fifty-three percent of 2- to 5-year-olds with ASD had at least one sleep problem, compared with $32 \%$ of controls [77]. Children with ASD often take more than 1 hour to fall asleep, and many have nocturnal awakenings that may last as long as 2-3 hours [78]. Sleep problems in ASD tend to persist past mid-puberty [79]. A longitudinal case-control study found that insomnia was 10 times more likely to be reported in ASD children than in controls, and that remission of sleep problems at 11 to 13 years was far less likely in ASD children than controls (8 versus 53\%) [79]. The most common sleep problems in ASD are sleep-onset delay, frequent nocturnal awakenings, and reduced sleep duration [80].

As for ADHD, as many as $70 \%$ of children with this disorder have been reported to display mild to severe sleep problems [81]. The prevalence rates differ by ADHD subtype, with the highest prevalence in the combined subtype [82], though sleepiness may be more frequent in the inattentive subtype [83]. In addition, psychiatric comorbidities and medication used both increase the prevalence of sleep problems in ADHD [82]. Children and/or their parents reported bedtime resistance, sleep-onset difficulties, night awakenings, difficulties with morning awakenings, sleep breathing problems, and daytime sleepiness significantly more than healthy controls [84]. Although there is no sleep problem specific to ADHD [81], the most commonly reported issue is "difficulty falling asleep [81]."

\subsubsection{Sleep Spindle Characteristics in Children with Intellec-} tual Disability. In 1962, E. L. Gibbs and F. A. Gibbs [85] reported the presence of "extreme spindles" in children with intellectual disability. Extreme spindles are characterized by their diffuse expression, much higher voltage (200-400 microvolts), and continuous occurrence. In addition, several studies examined sleep spindles in children with intellectual disability related to different genetic disorders [86]. These disorders included the neuronal ceroid lipofuscinosis (NCLs) [87] and Costello syndrome. The NCLs are a group of inherited progressive neurodegenerative lysosomal-storage disorders that are characterized by progressive intellectual and motor deterioration, seizures, and early death resulting from neural loss and widespread accumulation of lipopigments within cellular compartments [86]. The incidence (affected persons per live newborns) was reported to be $1: 12,500$ in the USA and Scandinavian countries, whereas the worldwide figure is $1: 100,000$ [88]. Children with NCLs were found to lack sleep spindles.

Costello syndrome, which is an autosomal dominant disorder caused by mutations in HRAS, has an estimated birth prevalence of $1: 300,000$ in the UK [89]. Costello Syndrome [90] is characterized by delayed development and intellectual disability, loose folds of skin, unusually flexible joints, and distinctive facial features including a large mouth. Researchers observed an increase in spindle amplitude (extreme spindles). Another study was conducted in children with malformations of cortical development [91]. These congenital brain disorders arise during embryonic and fetal development and are characterized by abnormalities in the volume, location, and/or architecture of cerebral gray and white matter. For each malformation subject, the authors identified a nonepileptic age- and sex-matched control patient for whom an EEG study had been performed within one year of the malformation subject's study, which was of the same type and demonstrated at least one sleep spindle burst. The authors found no difference between cases and controls in the mean spindle density or mean maximum spindle frequency, but significant between-group differences were seen in the laterality and anatomical distribution of spindles. Malformation subjects had a significantly higher proportion of unilateral sleep spindles compared to controls. Furthermore, although the sample sizes were small, subjects with unilateral malformations appeared to demonstrate a skewing of unilateral spindles toward the contralateral side, with fewer spindle bursts on the ipsilateral side to the malformation. Finally, malformation subjects had a significantly different overall anatomical distribution of sleep spindles, with an increased proportion of both anterior and diffuse spindles. Finally, Shibagaki et al. [92] reported a high prevalence of children with malformations of cortical development with no spindles, low incidence of sleep spindles, and/or extreme spindles.

\subsubsection{Sleep Spindles and Intellectual Performance in Children} with Intellectual Disability. In a series of studies [92-94], Shibagaki et al. classified the participants' levels of functioning according to their developmental quotients (DQs). They used the Tsumori and Inage questionnaire for infants and children, which divides the children into severe, moderate, and mildly intellectually disabled groups. They included children with intellectual disability associated with a range of disorders (e.g., congenital cerebral dysplasia, hydrocephaly, Rubinstein-Taybi syndrome, Down's syndrome, and chromosomal abnormality) and performed several studies investigating the occurrences of sleep spindles in these children. They found that children with intellectual disability frequently have no sleep spindles and that these children tended to have a lower DQ than those with intellectual disability with sleep spindles [94]. They reported a significant increase in abnormal clinical EEGs and a significant decrease in the DQs of children with high occurrences of shorter or no sleep spindles compared to those with higher occurrences of longer sleep spindles $[93,95]$.

\subsubsection{Sleep Spindles and Memory Consolidation in Children} with Intellectual Disability. No study has yet been reported that assessed memory consolidation in children with intellectual deficits.

Collectively, these studies show that children with intellectual disabilities due to a variety of heterogeneous genetic and developmental disorders show significant alterations in sleep spindles, including few or no sleep spindles, extreme spindles, and/or an increased proportion of unilateral spindles. In addition, the degree of sleep spindle abnormality is associated with the severity of cognitive impairment. In conditions with progressive neurodegeneration, spindle loss has been correlated with the state of the disease, with a complete absence of spindles seen during the most severe stages. 
3.2. Sleep Spindles in Children with Autism Spectrum Disorders (ASDs). ASDs are neurodevelopmental disorders characterized by repetitive behaviors and deficits in social interaction and communication. A diagnosis of ASD is made based on a constellation of requisite behavioral symptoms, including persistent deficits in social communication and interaction across multiple contexts, as well as restricted and repetitive patterns of behavior, interests, and activities.

3.2.1. Sleep Spindle Characteristics in Children with ASD. Studies comparing children/adolescents with ASD versus controls showed that the former had a lower sleep spindle density (Godbout et al. [96]), fewer sleep spindles over the central regions [97], a lower NREM stage 2 sleep spindle density in the prefrontal area, and shorter sleep spindle duration in the frontal area [98]. However, another study failed to find any difference in sleep spindles when comparing children with ASD and typically developing children [99].

\subsubsection{Sleep Spindles and Intellectual Performance in Children} with ASD. Reference [98] examined the associations between IQ (measured by the WISC-III) and sleep spindle density or duration in children with high functioning autism compared to typically developing children. The authors found that verbal IQ was negatively correlated with frontal spindle density and positively correlated with central spindle duration in typically developing children, whereas verbal IQ and the full scale IQ were negatively correlated with central spindle density in the ASD group.

3.2.3. Sleep Spindles and Memory Consolidation in Children with ASD. One recent study [99] examined memory consolidation in children with ASD. Twenty-two participants with ASD and 20 control participants between 9 and 16 years of age were trained to criterion on a spatial declarative memory task and then given a cued recall test. The subjects were allowed a period of daytime wake (Wake) or a night of sleep (Sleep), both of which were monitored with home-based polysomnography. Upon retest, better memory consolidation was observed in the Sleep group compared to the Wake group for both ASD and control children; however, participants with ASD had poorer overall memory consolidation. The change in performance across sleep, independent of medication and age, showed no significant relationship with any specific sleep parameter other than the total sleep time, and there was a trend toward less forgetting in the control group.

In summary, there are conflicting findings with respect to sleep spindles in children with ASD. Whereas the data from one research group suggest that individuals with ASD have shorter spindle durations, lower spindle density, and decreased sleep spindle frequencies, other groups have not replicated these findings. In addition, children with ASD have poorer memory consolidation than controls.

3.3. Sleep Spindles in Children with ADHD. Attention deficit/ hyperactivity disorder (ADHD) is one of the most common NDDs in childhood, affecting approximately $3 \%-5 \%$ of school-aged children [1] and enduring throughout adolescence and adulthood. A diagnosis of ADHD is dependent on developmentally inappropriate symptoms of inattention, hyperactivity, and/or impulsivity, with onset before the age of 7 years and impaired functioning in two or more settings [68].

3.3.1. Sleep Spindle Characteristics in Children with ADHD. There are conflicting findings with respect to sleep spindle characteristics in children with ADHD. Some studies [100] failed to find any statistically significant difference in the number of sleep spindles between hyperactive and control children, while other studies found significantly fewer [101] or more [102] sleep spindles in the EEGs of unmedicated hyperactive boys compared to normal controls.

3.3.2. Sleep Spindles and Intellectual Performance in Children with $A D H D$. No published study has yet examined this association.

At present, there is no consistent evidence for abnormal or altered sleep spindle activity in children with ADHD and sleep spindles activity per se has not been measured in studies examining memory consolidation of children with ADHD. However, there appear to be differences in the memory consolidation of children with ADHD versus typically developing children $[103,104]$, in that sleep benefits declarative memory in typically developing children, whereas ADHD children show deficits in sleep-associated consolidation of declarative memory and a reduced functionality of slow oscillations in this consolidation. In contrast, although procedural memory in typically developing children does not benefit from sleep, the data suggest that sleep appears to normalize the daytime deficits in procedural memory found among ADHD children [104].

3.4. Sleep Spindles in Children with Dyslexia. Developmental dyslexia is a hereditary neurological disorder that is characterized by the presence of severe and persistent reading and/or spelling impairments despite normal intelligence and adequate schooling. Almost no studies are available on sleep in children with dyslexia. The existing data have shown an alteration of sleep architecture characterized by an increase in slow wave sleep (SWS), a decrease in REM sleep, and a longer REM sleep latency in children with reading disabilities compared to controls [105].

One study [106] examined sleep spindles in 19 children with developmental dyslexia and 11 normally reading children between 7 and 16 years of age. The authors observed increases in spindle activity and sigma power in children with dyslexia and found that these parameters were correlated with the degree of dyslexic impairment. No information is available regarding sleep spindles and memory consolidation in this population. However, these results suggest that sleep spindle abnormalities may exist in children with dyslexia and that these abnormalities could be related to or correlated with impairment. Additional studies are needed to test these hypotheses.

\section{Discussion}

The goal of this paper is to review and integrate the available evidence regarding sleep spindle characteristics in children 
with NDD and (when possible) their associations with cognitive function. Before we attempt to integrate the findings across different disorders, we must note the significant methodological limitations of the existing work. Most studies had small sample sizes and thus might have overestimated the effect size and/or be difficult to replicate. This becomes even more challenging when researchers used data from the same participants in multiple publications (e.g., $[94,107])$. Another limitation involves the heterogeneity of the samples. First, clinical heterogeneity is inherent with each of the NDD under discussion. In addition, many of the studies may have suffered from developmental heterogeneity, as they lumped together participants of different developmental stages in terms of their puberty, sleep, and cognition. Heterogeneity may also have arisen from the inclusion of participants having different intelligence levels and/or comorbid conditions. These sources of heterogeneity cause us to question whether the results could be generalized to other settings and situations. Additional issues are related to methodological differences between the studies, which make it difficult to compare results directly. For example, in some studies EEG patterns were recorded during a full night of sleep, while in others sleep was induced by chloral hydrate during the daytime and only sleep recordings from routine clinical EEG were analyzed. In the latter case, medication effect cannot be ruled out, and the amount of time spent in stage 2 NREM sleep was limited, meaning that fewer sleep spindles were detected.

These noted limitations may be inherent challenges of investigating sleep spindles and cognition in children with NDD. It could be difficult to obtain larger, more homogenous groups given the prevalence of the disorders and their clinical nature which includes significant comorbidity and diversity. Technically and financially, it is challenging to conduct laboratory-based sleep studies in children who are challenging to manage, often have difficulty tolerating electrodes, may dislike being in an unfamiliar environment, and can resist cognitive testing. Hence, practical issues pose real barriers for the feasibility of large, homogenous studies that use objective measures of sleep and cognition.

Our review integrates the existing data pertaining to sleep spindle characteristics in children with NDDs and examines the results from studies seeking to correlate these differences with cognitive processes. Table 1 presents the evidence we reviewed regarding sleep spindles in children with NDD and in typically developing children. Several studies have found lower spindle density $[96-98,108]$ and extreme spindles [96] in children with ASD. In children with intellectual disabilities, absence of sleep spindles [87, 93, 107], extreme spindles [85], increased spindle activity [90], unilateral sleep spindles [91], and higher ratio of children with long spindles [95] have been documented. In children with dyslexia, increase in power of frequency bands $0.5-3 \mathrm{~Hz}$ and $11-12 \mathrm{~Hz}$ during $\mathrm{N} 2$ have been found [106]. This observed phenotypic variability neither proves nor refutes the existence of shared mechanisms in NDDs. However, shared molecular mechanisms have been shown to operate across disorder boundaries. It has also been suggested that, in the future, NDDs may be defined as pathological deviation of specific developmental processes and/or be seen to represent stages on a continuum of neurodevelopmental causality $[109,110]$. Although it has not been experimentally demonstrated, it is possible that alterations of sleep spindles may interfere with cognitive processes and behavior. Alternatively, it is possible that a proportion of neurodevelopmental impairments and sleep spindle alterations arise as independent manifestations of an underlying brain abnormality.

Even given the abovementioned issues, certain factors could be better controlled to meaningfully decrease the heterogeneity of the studies, thereby improving their relevance, validity, and generalizability. For example, better control of age and sex would allow future studies to focus on groups that are homogenous in these parameters. More studies might be required to cover all age groups for both sexes, but the information obtained in each study (even using small sample sizes) will be more significant. This will improve reproducibility and prevent findings which lack reliability.

\subsection{Potential Mechanisms Underlying the Interplay between} Sleep Spindles and Cognition in Children with Neurodevelopmental Disorders. One hypothesis regarding the nature of the association between sleep spindle characteristics and NDD is that impaired spindle activity could both reflect an abnormal neurodevelopmental trajectory and compromise the establishment of normal cognitive processes in this population. Although we do not yet know which pathways are involved or whether they are common to the various NDD, different hypotheses have been put forward regarding brain-related, genetic, and environmental influences. We will discuss these hypotheses in the context of the potential association between sleep spindles and NDD.

Many neurodevelopmental disorders (e.g., ADHD, ASD, and dyslexia) are accompanied by distinctive patterns of gray and white matter changes in the brain [111]. The evidence suggests that changes in gray matter may reflect structural changes in synapses and their dendrites, whereas those in the white matter reflect changes in myelination due to oligodendrocyte pathology. The presence of structural pathologies during development appears to provide a coherent biological model for the onset and course of NDD, while also suggesting a possible mechanistic basis for the associations between sleep spindles and cognitive abnormalities in such conditions $[32,112]$.

One factor that might facilitate the synchronization of neuronal networks and boost oscillatory activity is the strength of the underlying connections (i.e., the integrity of the white matter) $[113,114]$.

Individual differences in spindles and slow waves reportedly depend on the white matter microstructure across distributed networks. Thus, sleep oscillation profiles reflect both the synaptic-level dynamics of the neuronal network and the localized microstructural properties of the white matter tracts that form its structural backbone. Indeed, diffusiontensor imaging revealed that the expression profiles of sleep slow waves and spindles are partially determined by the axial diffusivity strength over long-range white matter tracts [115]. In contrast, the higher-frequency waves (including the beta and gamma frequency bands) mostly reflect short-distance synchronization, which increases during early development 
and might involve short-range white matter axons rather than the long-range white matter tracts. Associations have been found between individual-level sleep spindling and white matter integrity [115] and continuing white matter development during late adolescence [116]. White matter abnormalities have been found in several NDD, including ADHD [117122] and ASD. In the latter case, longitudinal data showed that white matter growth was slower among boys with autism, especially in the parietal lobes, [123] and diffusion imaging studies have revealed widespread disruption of white matter tracts in ASD patients, especially between regions implicated in social behavior [124-126]. Abnormal white matter development might, therefore, be a mechanism that cuts across neurodevelopmental disorders and might be related to both sleep spindles and disorders of cognitive function.

With respect to gray matter, the developmental changes in the prefrontal cortex differ depending on the IQ of the subject. The peak prefrontal gray matter volume is reportedly reached at 13 years in very high-IQ subjects, compared to 9 years in children of average IQ, with both groups reaching the same volume in late adolescence [32]. The growth and retraction of dendrites are dependent on the number and activity of the synapses that abut on their dendritic spines. Thus, changes in the gray matter volume are likely to reflect the formation and regression of synapses [127, 128]. The emergence of childhood ASD, ADHD, and dyslexia all involve divergence from the normal trajectories of gray matter development in different lobes of the brain (for review see [111]).

For sleep spindles, gray matter volume in the auditory and insular cortices was negatively correlated with sleep spindle frequency and found to be predictive of slower sleep spindles across individuals. Moreover, interindividual variability in sleep spindles over the sensory cortex was shown to predict the extent to which the sleep state is protected from experimental auditory stimulation [129]. These associations between slower sleep spindle frequency and gray matter volume in sensory areas may reflect the role of sleep spindles (of slower frequency) in protecting sleep against sensory disruption.

The association between sleep spindle frequency and the gray matter volume in the insular cortex provides a speculative basis for uniting various disparate findings in NDD. The loss of gray matter in NDD could result in abnormal sleep spindle activity, increasing the susceptibility to interference and thereby hindering sleep-dependent information processing. Future studies are needed to empirically test this possibility. Studies are also needed to examine the functional outcomes of abnormal white or gray matter volumes with respect to specific spindle characteristics, such as low frequency or higher density. Such associations could potentially explain a broad collection of cooccurring cognitive deficits in the NDD that are characterized by gray matter abnormalities (e.g., ASD, ADHD, and various learning disabilities).

4.2. Clinical Implications and Future Directions. This review indicates that future studies should continue examining sleep spindle characteristics and their associations with cognition in children with NDD. This is important because it is possible that aberrant spindle activity cuts across a number of physiological and pathological conditions, potentially reflecting impairments in neuroplasticity across these conditions. The examination of spindle measures in children with NDD could uncover developmental alterations that may characterize progression of these disorders. Furthermore, since sleep has been demonstrated to improve memory consolidation and learning, potential changes in sleep spindles may also be relevant to our complete understanding of the cognitive difficulties observed in many patients with NDD. Future studies should seek to use large, well-phenotyped samples that are homogeneous in age and sex and represent all developmental stages of the disorder being investigated. They should also use objective and standardized measures of sleep and cognition plus, when possible, experimental designs that will allow the authors to establish causality. Such studies would significantly advance our understanding of this topic and could move us toward answering important questions, such as what does it mean to have fewer or aberrant spindles, in terms of cognitive functioning? Can sleep spindle assessment in infancy and toddlerhood enable the early identification of learning or neurocognitive disabilities that could be addressed at an early age (e.g., ADHD or dyslexia), potentially reducing their negative impact on the developmental trajectory of atrisk children? Longitudinal studies examining sleep spindle characteristics and later outcomes in children at risk for NDD could begin to answer these questions and potentially identify reliable markers that could be used for routine sleep assessments of infants and toddlers. This could open the door to practical and relatively inexpensive methods for the early identification and prevention of potentially lifelong disorders that are debilitating yet treatable (e.g., dyslexia or ADHD).

Given the findings that implicate sleep spindles in memory consolidation and brain plasticity, another important question is whether it could be possible to stimulate sleep spindle activity as a means to improve cognition in children with NDD. Since little or no spindle activity is associated with poorer memory consolidation and/or intellectual performance, could the stimulation of sleep spindles or other oscillations during sleep increase brain plasticity, improve memory consolidation, and/or improve intellectual performance? One study conducted in children with ADHD provided promising initial evidence by suggesting that this may be the case; external enhancement of frontal slow oscillations (SO) at $0.75 \mathrm{~Hz}$ by transcranial oscillating direct current stimulation (DCS) was shown to elevate sleep-dependent memory in children with ADHD to the level of healthy controls. While children with ADHD showed worse memory performance than healthy controls when subjected to the sham condition, this memory deficit vanished following the application of DCS during sleep. Several other methods that have been shown to increase sleep spindle activity or slow wave activity during subsequent sleep have also improved sleep-dependent memory consolidation, including anodal transcranial DCS $[130,131]$, intensive physical exercise during the daytime [132], neurofeedback [133, 134], and pharmacological manipulation of spindle density [135]. Future studies should therefore investigate whether enhancement of sleep spindles through these or other means could improve 
sleep-dependent memory consolidation or other aspects of cognitive function in children with NDD.

In summary, relatively few studies have provided detailed examination of sleep spindle characteristics in children with NDD and their associations with cognitive function. Existing studies suffer from the use of multiple, often nonstandardized, methodologies and lack of exploration across the full range of the NDD from early to late stages. Despite these significant limitations, there is collective evidence that investigation of sleep spindles in children with and without NDD is important because the mechanisms that underlie spindle generation are involved with plasticity and with stabilization of sleep, both of which are known to be important in supporting optimal cognitive development and function.

\section{Competing Interests}

The authors declare that they have no competing interests.

\section{References}

[1] S. Goldstein and C. R. Reynolds, Handbook of Neurodevelopmental and Genetic Disorders in Children, 2/e, Guilford Press, 2010.

[2] Z. A. Cope, S. B. Powell, and J. W. Young, "Modeling neurodevelopmental cognitive deficits in tasks with cross-species translational validity," Genes, Brain and Behavior, vol. 15, no. 1, pp. 27-44, 2016.

[3] J. M. Berkman, "Sleep in children with developmental disabilities," Medicine and Health, Rhode Island, vol. 89, no. 3, pp. 9496, 2006.

[4] S. M. Doran, M. T. Harvey, and R. H. Horner, "Sleep and developmental disabilities: assessment, treatment, and outcome measures," Mental Retardation, vol. 44, no. 1, pp. 13-27, 2006.

[5] M. H. Silber, S. Ancoli-Israel, M. H. Bonnet et al., "The visual scoring of sleep in adults," Journal of Clinical Sleep Medicine, vol. 3, no. 2, pp. 121-131, 2007.

[6] L. De Gennaro and M. Ferrara, "Sleep spindles: an overview," Sleep Medicine Reviews, vol. 7, no. 5, pp. 423-440, 2003.

[7] L. V. Marcuse, M. C. Fields, and J. J. Yoo, Rowan's Primer of EEG, Elsevier Health Sciences, 2015.

[8] M. Mölle, T. O. Bergmann, L. Marshall, and J. Born, "Fast and slow spindles during the sleep slow oscillation: disparate coalescence and engagement in memory processing," Sleep, vol. 34, no. 10, pp. 1411-1421, 2011.

[9] M. Schabus, T. T. Dang-Vu, G. Albouy et al., "Hemodynamic cerebral correlates of sleep spindles during human non-rapid eye movement sleep," Proceedings of the National Academy of Sciences of the United States of America, vol. 104, no. 32, pp. 13164-13169, 2007.

[10] T. Andrillon, Y. Nir, R. J. Staba et al., "Sleep spindles in humans: Insights from intracranial EEG and unit recordings," The Journal of Neuroscience, vol. 31, no. 49, pp. 17821-17834, 2011.

[11] L. Peter-Derex, J.-C. Comte, F. Mauguière, and P. A. Salin, "Density and frequency caudo-rostral gradients of sleep spindles recorded in the human cortex," Sleep, vol. 35, no. 1, pp. 69-79, 2012.

[12] D. Contreras, A. Destexhe, and M. Steriade, "Spindle oscillations during cortical spreading depression in naturally sleeping cats," Neuroscience, vol. 77, no. 4, pp. 933-936, 1997.
[13] W. R. Jankel and E. Niedermeyer, "Sleep spindles," Journal of Clinical Neurophysiology, vol. 2, no. 1, pp. 1-35, 1985.

[14] Y. Urakami, A. A. Ioannides, and G. K. Kostopoulos, "Sleep spindles-as a biomarker of brain function and plasticity," in Advances in Clinical Neurophysiology, chapter 4, InTech, 2012.

[15] S. Shinomiya, K. Nagata, K. Takahashi, and T. Masumura, "Development of sleep spindles in young children and adolescents," Clinical EEG Electroencephalography, vol. 30, no. 2, pp. 39-43, 1999.

[16] M. Steriade, D. A. McCormick, and T. J. Sejnowski, “Thalamocortical oscillations in the sleeping and aroused brain," Science, vol. 262, no. 5134, pp. 679-685, 1993.

[17] M. Steriade and R. McCarley, Brain Control of Waking and Sleep, Klüver-Plenum, New York, NY, USA, 2005.

[18] J. Louis, J. X. Zhang, M. Revol, G. Debilly, and M. J. Challamel, "Ontogenesis of nocturnal organization of sleep spindles: a longitudinal study during the first 6 months of life," Electroencephalography and Clinical Neurophysiology, vol. 83, no. 5, pp. 289-296, 1992.

[19] A. Nicolas, D. Petit, S. Rompré, and J. Montplaisir, "Sleep spindle characteristics in healthy subjects of different age groups," Clinical Neurophysiology, vol. 112, no. 3, pp. 521-527, 2001.

[20] O. G. Jenni, A. A. Borbély, and P. Achermann, "Development of the nocturnal sleep electroencephalogram in human infants," American Journal of Physiology-Regulatory, Integrative and Comparative Physiology, vol. 286, no. 3, pp. R528-R538, 2004.

[21] J. E. Stockard-Pope, S. S. Werner, and R. G. Bickford, Atlas of Neonatal Electroencephalography, Raven Press, 1992.

[22] D. R. Metcalf, "The effect of extrauterine experience on the ontogenesis of EEG sleep spindles," Psychosomatic Medicine, vol. 31, no. 5, pp. 393-399, 1969.

[23] D. R. Metcalf and K. Jordan, "EEG ontogenesis in normal children," in Drugs, Development, and Cerebral Function, pp. 127-128, Springfield'Chas. Thomas, 1972.

[24] R. J. Ellingson and J. F. Peters, "Development of EEG and daytime sleep patterns in normal full-term infants during the first 3 months of life: longitudinal observations," Electroencephalography and Clinical Neurophysiology, vol. 49, no. 1-2, pp. 112-124, 1980.

[25] P. Kellaway, "An orderly approach to visual analysis: characteristics of the normal EEG of adults and children," Current Practice of Clinical Electroencephalography, vol. 2, pp. 139-199, 1990.

[26] P. E. Tanguay, E. M. Ornitz, A. Kaplan, and E. S. Bozzo, "Evolution of sleep spindles in childhood," Electroencephalography and Clinical Neurophysiology, vol. 38, no. 2, pp. 175-181, 1975.

[27] S. Scholle, G. Zwacka, and H. C. Scholle, "Sleep spindle evolution from infancy to adolescence," Clinical Neurophysiology, vol. 118, no. 7, pp. 1525-1531, 2007.

[28] N. Dehghani, S. S. Cash, and E. Halgren, "Topographical frequency dynamics within EEG and MEG sleep spindles," Clinical Neurophysiology, vol. 122, no. 2, pp. 229-235, 2011.

[29] O. G. Jenni and M. A. Carskadon, "Spectral analysis of the sleep electroencephalogram during adolescence," Sleep, vol. 27, no. 4, pp. 774-783, 2004.

[30] S. Kurth, M. Ringli, A. Geiger, M. LeBourgeois, O. G. Jenni, and R. Huber, "Mapping of cortical activity in the first two decades of life: a high-density sleep electroencephalogram study," Journal of Neuroscience, vol. 30, no. 40, pp. 13211-13219, 2010.

[31] P. Shaw, D. Greenstein, J. Lerch et al., "Intellectual ability and cortical development in children and adolescents," Nature, vol. 440, no. 7084, pp. 676-679, 2006. 
[32] N. Gogtay and P. M. Thompson, "Mapping gray matter development: implications for typical development and vulnerability to psychopathology," Brain and Cognition, vol. 72, no. 1, pp. 6-15, 2010.

[33] I. Feinberg and I. G. Campbell, "Sleep EEG changes during adolescence: an index of a fundamental brain reorganization," Brain and Cognition, vol. 72, no. 1, pp. 56-65, 2010.

[34] H. G. Schnack, N. E. M. Van Haren, R. M. Brouwer et al., "Changes in thickness and surface area of the human cortex and their relationship with intelligence," Cerebral Cortex, vol. 25, no. 6, pp. 1608-1617, 2015.

[35] V. V. Vyazovskiy, U. Olcese, Y. M. Lazimy et al., "Cortical firing and sleep homeostasis," Neuron, vol. 63, no. 6, pp. 865-878, 2009.

[36] C. Lebel, L. Walker, A. Leemans, L. Phillips, and C. Beaulieu, "Microstructural maturation of the human brain from childhood to adulthood," NeuroImage, vol. 40, no. 3, pp. 1044-1055, 2008.

[37] M. Kharitonova, R. E. Martin, J. D. E. Gabrieli, and M. A. Sheridan, "Cortical gray-matter thinning is associated with agerelated improvements on executive function tasks," Developmental Cognitive Neuroscience, vol. 6, pp. 61-71, 2013.

[38] F. Lopes da Silva and A. Van Rotterdam, "Biophysical aspects of EEG and MEG generation," in Electroencephalography: Basic Principles, Clinical Applications and Related Fields, E. Niedermeyer and F. Lopes da Silva, Eds., Urban \& Schwarzenberg, Munich, Germany, 1982.

[39] L. Tarokh and M. A. Carskadon, "Developmental changes in the human sleep EEG during early adolescence," Sleep, vol. 33, no. 6, pp. 801-809, 2010.

[40] I. Feinberg, E. De Bie, N. M. Davis, and I. G. Campbell, “Topographic differences in the adolescent maturation of the slow wave EEG during NREM sleep," Sleep, vol. 34, no. 3, pp. 325333, 2011.

[41] I. G. Campbell, N. Darchia, W. Y. Khaw, L. M. Higgins, and I. Feinberg, "Sleep EEG evidence of sex differences in adolescent brain maturation," Sleep, vol. 28, no. 5, pp. 637-643, 2005.

[42] I. G. Campbell and I. Feinberg, "Longitudinal trajectories of non-rapid eye movement delta and theta EEG as indicators of adolescent brain maturation," Proceedings of the National Academy of Sciences of the United States of America, vol. 106, no. 13, pp. 5177-5180, 2009.

[43] I. Feinberg, J. D. March, K. Flach, T. Maloney, W.-J. Chern, and F. Travis, "Maturational changes in amplitude, incidence and cyclic pattern of the 0 to $3 \mathrm{~Hz}$ (Delta) electroencephalogram of human sleep," Brain Dysfunction, vol. 3, no. 3-4, pp. 183-192, 1990.

[44] J. W. Bang, O. Khalilzadeh, M. Hämäläinen, T. Watanabe, and Y. Sasaki, "Location specific sleep spindle activity in the early visual areas and perceptual learning," Vision Research, vol. 99, pp. 162-171, 2014.

[45] R. Bódizs, T. Kis, A. S. Lázár et al., "Prediction of general mental ability based on neural oscillation measures of sleep," Journal of Sleep Research, vol. 14, no. 3, pp. 285-292, 2005.

[46] C. Lustenberger, A. Maric, R. Dür, P. Achermann, and R. Huber, "Triangular relationship between sleep spindle activity, general cognitive ability and the efficiency of declarative learning," PLoS ONE, vol. 7, no. 11, Article ID e49561, 2012.

[47] R. Cox, W. F. Hofman, M. de Boer, and L. M. Talamini, "Local sleep spindle modulations in relation to specific memory cues," NeuroImage, vol. 99, pp. 103-110, 2014.
[48] T. T. Dang-Vu, S. M. McKinney, O. M. Buxton, J. M. Solet, and J. M. Ellenbogen, "Spontaneous brain rhythms predict sleep stability in the face of noise," Current Biology, vol. 20, no. 15, pp. R626-R627, 2010.

[49] S. M. Sherman, "Tonic and burst firing: dual modes of thalamocortical relay," Trends in Neurosciences, vol. 24, no. 2, pp. 122126, 2001.

[50] R. Stickgold, “Sleep-dependent memory consolidation," Nature, vol. 437, no. 7063, pp. 1272-1278, 2005.

[51] C. Pavlides and J. Winson, "Influences of hippocampal place cell firing in the awake state on the activity of these cells during subsequent sleep episodes," Journal of Neuroscience, vol. 9, no. 8, pp. 2907-2918, 1989.

[52] S. Fischer, M. Hallschmid, A. L. Elsner, and J. Born, "Sleep forms memory for finger skills," Proceedings of the National Academy of Sciences of the United States of America, vol. 99, no. 18, pp. 11987-11991, 2002.

[53] Y. Yotsumoto, Y. Sasaki, P. Chan et al., "Location-specific cortical activation changes during sleep after training for perceptual learning," Current Biology, vol. 19, no. 15, pp. 1278-1282, 2009.

[54] S. Gais and J. Born, "Declarative memory consolidation: mechanisms acting during human sleep," Learning \& Memory, vol. 11, no. 6, pp. 679-685, 2004.

[55] I. Wilhelm, S. Diekelmann, and J. Born, "Sleep in children improves memory performance on declarative but not procedural tasks," Learning and Memory, vol. 15, no. 5, pp. 373-377, 2008.

[56] S. Fischer, I. Wilhelm, and J. Born, “Developmental differences in sleep's role for implicit off-line learning: comparing children with adults," Journal of Cognitive Neuroscience, vol. 19, no. 2, pp. 214-227, 2007.

[57] I. Timofeev, F. Grenier, M. Bazhenov, A. R. Houweling, T. J. Sejnowski, and M. Steriade, "Short and medium term plasticity associated with augmenting responses in cortical slabs and spindles in intact cortex of cats in vivo," The Journal of Physiology, vol. 542, no. 2, pp. 583-598, 2002.

[58] S. M. Fogel and C. T. Smith, "The function of the sleep spindle: a physiological index of intelligence and a mechanism for sleep-dependent memory consolidation," Neuroscience \& Biobehavioral Reviews, vol. 35, no. 5, pp. 1154-1165, 2011.

[59] D. Contreras, A. Destexhe, and M. Steriade, "Intracellular and computational characterization of the intracortical inhibitory control of synchronized thalamic inputs in vivo," Journal of Neurophysiology, vol. 78, no. 1, pp. 335-350, 1997.

[60] B. Rasch and J. Born, "About sleep's role in memory," Physiological Reviews, vol. 93, no. 2, pp. 681-766, 2013.

[61] A. Geiger, R. Huber, S. Kurth, M. Ringli, O. G. Jenni, and P. Achermann, "The sleep EEG as a marker of intellectual ability in school age children," Sleep, vol. 34, no. 2, pp. 181-189, 2011.

[62] R. Gruber, M. S. Wise, S. Frenette et al., "The association between sleep spindles and IQ in healthy school-age children," International Journal of Psychophysiology, vol. 89, no. 2, pp. 229240, 2013.

[63] A. Chatburn, S. Coussens, K. Lushington, D. Kennedy, M. Baumert, and M. Kohler, "Sleep spindle activity and cognitive performance in healthy children," Sleep, vol. 36, no. 2, pp. 237243, 2013.

[64] K. Hoedlmoser, D. P. J. Heib, J. Roell et al., "Slow sleep spindle activity, declarative memory, and general cognitive abilities in children," Sleep, vol. 37, no. 9, pp. 1501-1512, 2014. 
[65] R. G. Astill, G. Piantoni, R. J. E. M. Raymann et al., "Sleep spindle and slow wave frequency reflect motor skill performance in primary school-age children," Frontiers in Human Neuroscience, vol. 8, article 910, 2014.

[66] R. Bódizs, F. Gombos, P. P. Ujma, and I. Kovács, “Sleep Spindling and Fluid intelligence across adolescent development: sex matters," Frontiers in Human Neuroscience, vol. 8, article 952, 2014.

[67] M. R. Doucette, S. Kurth, N. Chevalier, Y. Munakata, and M. LeBourgeois, "Topography of slow sigma power during sleep is associated with processing speed in preschool children," Brain Sciences, vol. 5, no. 4, pp. 494-508, 2015.

[68] American Psychiatric Association, Diagnostic and Statistical Manual of Mental Disorders (DSM-5), American Psychiatric Association Publishing, Arlington, Va, USA, 2013.

[69] J. A. Owens and J. A. Mindell, "Pediatric insomnia," Pediatric Clinics of North America, vol. 58, no. 3, pp. 555-569, 2011.

[70] O. S. Ipsiroglu, W. H. McKellin, N. Carey, and C. Loock, “'They silently live in terror...' why sleep problems and night-time related quality-of-life are missed in children with a fetal alcohol spectrum disorder," Social Science \& Medicine, vol. 79, no. 1, pp. 76-83, 2013.

[71] L. Quine, "Sleep problems in children with mental handicap," Journal of Mental Deficiency Research, vol. 35, no. 4, pp. 269290, 1991.

[72] L. Wiggs and G. Stores, "Sleep problems in children with severe intellectual disabilities: what help is being provided?" Journal of Applied Research in Intellectual Disabilities, vol. 9, no. 2, pp. 160-165, 1996.

[73] L. B. Bartlett, V. Rooney, and S. Spedding, "Nocturnal difficulties in a population of mentally handicapped children," The British Journal of Mental Subnormality, vol. 31, no. 60, pp. 54-59, 1985.

[74] A. L. Richdale, "Sleep problems in autism: prevalence, cause, and intervention," Developmental Medicine \& Child Neurology, vol. 41, no. 1, pp. 60-66, 1999.

[75] B. A. Malow, M. L. Marzec, S. G. McGrew, L. Wang, L. M. Henderson, and W. L. Stone, "Characterizing sleep in children with autism spectrum disorders: a multidimensional approach," Sleep, vol. 29, no. 12, pp. 1563-1571, 2006.

[76] L. J. Meltzer, "Brief report: sleep in parents of children with autism spectrum disorders," Journal of Pediatric Psychology, vol. 33, no. 4, pp. 380-386, 2008.

[77] P. Krakowiak, B. Goodlin-Jones, I. Hertz-Picciotto, L. A. Croen, and R. L. Hansen, "Sleep problems in children with autism spectrum disorders, developmental delays, and typical development: a population-based study," Journal of Sleep Research, vol. 21, no. 2, p. 231, 2012.

[78] F. Cortesi, F. Giannotti, A. Ivanenko, and K. Johnson, "Sleep in children with autistic spectrum disorder," Sleep Medicine, vol. 11, no. 7, pp. 659-664, 2010.

[79] B. Sivertsen, M.-B. Posserud, C. Gillberg, A. J. Lundervold, and M. Hysing, "Sleep problems in children with autism spectrum problems: a longitudinal population-based study," Autism, vol. 16, no. 2, pp. 139-150, 2012.

[80] S. Kotagal and E. Broomall, "Sleep in children with autism spectrum disorder," Pediatric Neurology, vol. 47, no. 4, pp. 242251, 2012.

[81] V. Sung, H. Hiscock, E. Sciberras, and D. Efron, "Sleep problems in children with attention-deficit/hyperactivity disorder: prevalence and the effect on the child and family," Archives of
Pediatrics \& Adolescent Medicine, vol. 162, no. 4, pp. 336-342, 2008.

[82] S. D. Mayes, S. L. Calhoun, E. O. Bixler et al., "ADHD subtypes and comorbid anxiety, depression, and oppositional-defiant disorder: differences in sleep problems," Journal of Pediatric Psychology, vol. 34, no. 3, pp. 328-337, 2009.

[83] P. Corkum, H. Moldofsky, S. Hogg-Johnson, T. Humphries, and R. Tannock, "Sleep problems in children with attentiondeficit/hyperactivity disorder: Impact of subtype, comorbidity, and stimulant medication," Journal of the American Academy of Child and Adolescent Psychiatry, vol. 38, no. 10, pp. 1285-1293, 1999.

[84] S. Cortese, S. V. Faraone, E. Konofal, and M. Lecendreux, "Sleep in children with attention-deficit/hyperactivity disorder: meta-analysis of subjective and objective studies," Journal of the American Academy of Child \& Adolescent Psychiatry, vol. 48, no. 9, pp. 894-908, 2009.

[85] E. L. Gibbs and F. A. Gibbs, "Extreme spindles: correlation of electroencephalographic sleep pattern with mental retardation," Science, vol. 138, no. 3545, pp. 1106-1107, 1962.

[86] M. Haltia, "The neuronal ceroid-lipofuscinoses: from past to present," Biochimica et Biophysica Acta (BBA)-Molecular Basis of Disease, vol. 1762, no. 10, pp. 850-856, 2006.

[87] E. Veneselli, R. Biancheri, S. Buoni, and A. Fois, "Clinical and EEG findings in 18 cases of late infantile neuronal ceroid lipofuscinosis," Brain and Development, vol. 23, no. 5, pp. 306-311, 2001.

[88] P. Santavuori, "Neuronal ceroid-lipofuscinoses in childhood," Brain and Development, vol. 10, no. 2, pp. 80-83, 1988.

[89] K. W. Gripp and A. E. Lin, "Costello syndrome: a Ras/mitogen activated protein kinase pathway syndrome (rasopathy) resulting from HRAS germline mutations," Genetics in Medicine, vol. 14, no. 3, pp. 285-292, 2012.

[90] G. D. Marca, C. Leoni, S. Dittoni et al., "Increased sleep spindle activity in patients with Costello syndrome (HRAS gene mutation)," Journal of Clinical Neurophysiology, vol. 28, no. 3, pp. 314-318, 2011.

[91] M. F. Selvitelli, K. B. Krishnamurthy, A. G. Herzog, D. L. Schomer, and B. S. Chang, "Sleep spindle alterations in patients with malformations of cortical development," Brain and Development, vol. 31, no. 2, pp. 163-168, 2009.

[92] M. Shibagaki, S. Kiyono, and T. Takeuchi, "Nocturnal sleep in infants with congenital cerebral malformation," Clinical EEG (Electroencephalography), vol. 17, no. 2, pp. 92-104, 1986.

[93] M. Shibagaki, S. Kiyono, and K. Watanabe, "Nocturnal sleep in severely mentally retarded children: abnormal EEG patterns in sleep cycle," Electroencephalography and Clinical Neurophysiology, vol. 49, no. 3-4, pp. 337-344, 1980.

[94] M. Shibagaki, S. Kiyono, K. Watanabe, and S. Hakamada, "Concurrent occurrence of rapid eye movement with spindle burst during nocturnal sleep in mentally retarded children," Electroencephalography and Clinical Neurophysiology, vol. 53, no. 1, pp. 27-35, 1982.

[95] M. Shibagaki and S. Kiyono, "Duration of spindle bursts during nocturnal sleep in mentally retarded children," Electroencephalography and Clinical Neurophysiology, vol. 55, no. 6, pp. 645-651, 1983.

[96] R. Godbout, C. Bergeron, É. Limoges, E. Stip, and L. Mottron, “A laboratory study of sleep in Asperger's syndrome," NeuroReport, vol. 11, no. 1, pp. 127-130, 2000. 
[97] É. Limoges, C. Bolduc, C. Berthiaume, L. Mottron, and R. Godbout, "Relationship between poor sleep and daytime cognitive performance in young adults with autism," Research in Developmental Disabilities, vol. 34, no. 4, pp. 1322-1335, 2013.

[98] S. Tessier, A. Lambert, M. Chicoine, P. Scherzer, I. Soulières, and R. Godbout, "Intelligence measures and stage 2 sleep in typically-developing and autistic children," International Journal of Psychophysiology, vol. 97, no. 1, pp. 58-65, 2015.

[99] K. Maski, H. Holbrook, D. Manoach, E. Hanson, K. Kapur, and R. Stickgold, "Sleep dependent memory consolidation in children with autism spectrum disorder," Sleep, vol. 38, no. 12, pp. 1955-1963, 2015.

[100] N. A. Kiesow and W. W. Surwillo, "Sleep spindles in the EEGs of hyperactive children," Psychological Reports, vol. 60, no. 1, pp. 139-144, 1987.

[101] A. Khan and A. Rechtschaffen, "Sleep patterns and sleep spindles in hyperkinetic children," Sleep Research, vol. 7, article 137, 1978.

[102] L. Poitras, F. W. Bylsma, J. Simeon, and R. T. Pivik, "Cortical sleep spindle activity in hyperkinetic children," Sleep Research, vol. 10, article 117, 1981.

[103] A. Prehn-Kristensen, R. Göder, J. Fischer et al., "Reduced sleepassociated consolidation of declarative memory in attentiondeficit/hyperactivity disorder," Sleep Medicine, vol. 12, no. 7, pp. 672-679, 2011.

[104] A. Prehn-Kristensen, I. Molzow, M. Munz et al., "Sleep restores daytime deficits in procedural memory in children with attention-deficit/hyperactivity disorder," Research in Developmental Disabilities, vol. 32, no. 6, pp. 2480-2488, 2011.

[105] L. Mercier, R. T. Pivik, and K. Busby, "Sleep patterns in reading disabled children," Sleep: Journal of Sleep Research \& Sleep Medicine, vol. 16, no. 3, pp. 207-215, 1993.

[106] O. Bruni, R. Ferri, L. Novelli et al., "Sleep spindle activity is correlated with reading abilities in developmental dyslexia," Sleep, vol. 32, no. 10, pp. 1333-1340, 2009.

[107] M. Shibagaki, S. Kiyono, and T. Takeuchi, "Nocturnal sleep in mentally retarded infants with cerebral palsy," Electroencephalography and Clinical Neurophysiology, vol. 61, no. 6, pp. 465-471, 1985.

[108] É. Limoges, L. Mottron, C. Bolduc, C. Berthiaume, and R. Godbout, "Atypical sleep architecture and the autism phenotype," Brain, vol. 128, no. 5, pp. 1049-1061, 2005.

[109] M. J. Owen, "Implications of genetic findings for understanding schizophrenia," Schizophrenia Bulletin, vol. 38, no. 5, pp. 904907, 2012.

[110] E. Kelleher and A. Corvin, Overlapping Etiology of Neurodevelopmental Disorders, The Genetics of Neurodevelopmental Disorders, 2015.

[111] M. R. Bennett and J. Lagopoulos, "Neurodevelopmental sequelae associated with gray and white matter changes and their cellular basis: a comparison between Autism Spectrum Disorder, ADHD and dyslexia," International Journal of Developmental Neuroscience, vol. 46, pp. 132-143, 2015.

[112] R. E. Roberts, E. J. Anderson, and M. Husain, "White matter microstructure and cognitive function," The Neuroscientist, vol. 19, no. 1, pp. 8-15, 2013.

[113] P. J. Uhlhaas, F. Roux, W. Singer, C. Haenschel, R. Sireteanu, and E. Rodriguez, "The development of neural synchrony reflects late maturation and restructuring of functional networks in humans," Proceedings of the National Academy of Sciences of the United States of America, vol. 106, no. 24, pp. 9866-9871, 2009.
[114] P. J. Uhlhaas and W. Singer, "The development of neural synchrony and large-scale cortical networks during adolescence: relevance for the pathophysiology of schizophrenia and neurodevelopmental hypothesis," Schizophrenia Bulletin, vol. 37, no. 3, pp. 514-523, 2011.

[115] G. Piantoni, S.-S. Poil, K. Linkenkaer-Hansen et al., "Individual differences in white matter diffusion affect sleep oscillations," The Journal of Neuroscience, vol. 33, no. 1, pp. 227-233, 2013.

[116] C. D. Ladouceur, J. S. Peper, E. A. Crone, and R. E. Dahl, "White matter development in adolescence: the influence of puberty and implications for affective disorders," Developmental Cognitive Neuroscience, vol. 2, no. 1, pp. 36-54, 2012.

[117] M. Ashtari, S. Kumra, S. L. Bhaskar et al., "Attention-deficit/ hyperactivity disorder: a preliminary diffusion tensor imaging study," Biological Psychiatry, vol. 57, no. 5, pp. 448-455, 2005.

[118] L. S. Hamilton, J. G. Levitt, J. O'Neill et al., "Reduced white matter integrity in attention-deficit hyperactivity disorder," $\mathrm{Neu}$ roReport, vol. 19, no. 17, pp. 1705-1708, 2008.

[119] M. N. Pavuluri, S. Yang, K. Kamineni et al., "Diffusion tensor imaging study of white matter fiber tracts in pediatric bipolar disorder and attention-deficit/hyperactivity disorder," Biological Psychiatry, vol. 65, no. 7, pp. 586-593, 2009.

[120] M.-G. Qiu, Z. Ye, Q.-Y. Li, G.-J. Liu, B. Xie, and J. Wang, "Changes of Brain structure and function in ADHD children," Brain Topography, vol. 24, no. 3-4, pp. 243-252, 2011.

[121] T. J. Silk, A. Vance, N. Rinehart, J. L. Bradshaw, and R. Cunnington, "Structural development of the basal ganglia in attention deficit hyperactivity disorder: a diffusion tensor imaging study," Psychiatry Research: Neuroimaging, vol. 172, no. 3, pp. 220-225, 2009.

[122] K. E. Lawrence, J. G. Levitt, S. K. Loo et al., "White matter microstructure in subjects with attention-deficit/hyperactivity disorder and their siblings," Journal of the American Academy of Child \& Adolescent Psychiatry, vol. 52, no. 4, pp. 431-440.e4, 2013.

[123] X. Hua, P. M. Thompson, A. D. Leow et al., "Brain growth rate abnormalities visualized in adolescents with autism," Human Brain Mapping, vol. 34, no. 2, pp. 425-436, 2013.

[124] N. Barnea-Goraly, H. Kwon, V. Menon, S. Eliez, L. Lotspeich, and A. L. Reiss, "White matter structure in autism: preliminary evidence from diffusion tensor imaging," Biological Psychiatry, vol. 55, no. 3, pp. 323-326, 2004.

[125] N. Barnea-Goraly, L. J. Lotspeich, and A. L. Reiss, "Similar white matter aberrations in children with autism and their unaffected siblings: a diffusion tensor imaging study using tract-based spatial statistics," Archives of General Psychiatry, vol. 67, no. 10, pp. 1052-1060, 2010.

[126] D. K. Shukla, B. Keehn, A. J. Lincoln, and R.-A. Müller, "White matter compromise of callosal and subcortical fiber tracts in children with autism spectrum disorder: a diffusion tensor imaging study," Journal of the American Academy of Child of Adolescent Psychiatry, vol. 49, no. 12, pp. 1269-1278.e2, 2010.

[127] M. R. Bennett, "The prefrontal-limbic network in depression: a core pathology of synapse regression," Progress in Neurobiology, vol. 93, no. 4, pp. 457-467, 2011.

[128] M. R. Bennett, "Schizophrenia: susceptibility genes, dendriticspine pathology and gray matter loss," Progress in Neurobiology, vol. 95, no. 3, pp. 275-300, 2011.

[129] T. T. Dang-Vu, M. Schabus, M. Desseilles et al., "Spontaneous neural activity during human slow wave sleep," Proceedings of the National Academy of Sciences of the United States of America, vol. 105, no. 39, pp. 15160-15165, 2008. 
[130] L. Marshall, H. Helgadóttir, M. Mölle, and J. Born, "Boosting slow oscillations during sleep potentiates memory," Nature, vol. 444, no. 7119, pp. 610-613, 2006.

[131] L. Marshall, M. Mölle, M. Hallschmid, and J. Born, “Transcranial direct current stimulation during sleep improves declarative memory," The Journal of Neuroscience, vol. 24, no. 44, pp. 9985-9992, 2004.

[132] M. Dworak, A. Wiater, D. Alfer, E. Stephan, W. Hollmann, and H. K. Strüder, "Increased slow wave sleep and reduced stage 2 sleep in children depending on exercise intensity," Sleep Medicine, vol. 9, no. 3, pp. 266-272, 2008.

[133] K. Hoedlmoser, T. Pecherstorfer, G. Gruber et al., "Instrumental conditioning of human sensorimotor rhythm $(12-15 \mathrm{~Hz})$ and its impact on sleep as well as declarative learning," Sleep, vol. 31, no. 10, pp. 1401-1408, 2008.

[134] M. B. Sterman, R. C. Howe, and L. R. Macdonald, "Facilitation of spindle-burst sleep by conditioning of electroencephalographic activity while awake," Science, vol. 167, no. 3921, pp. 1146-1148, 1970.

[135] S. C. Mednick, E. A. McDevitt, J. K. Walsh et al., "The critical role of sleep spindles in hippocampal-dependent memory: a pharmacology study," The Journal of Neuroscience, vol. 33, no. 10, pp. 4494-4504, 2013. 

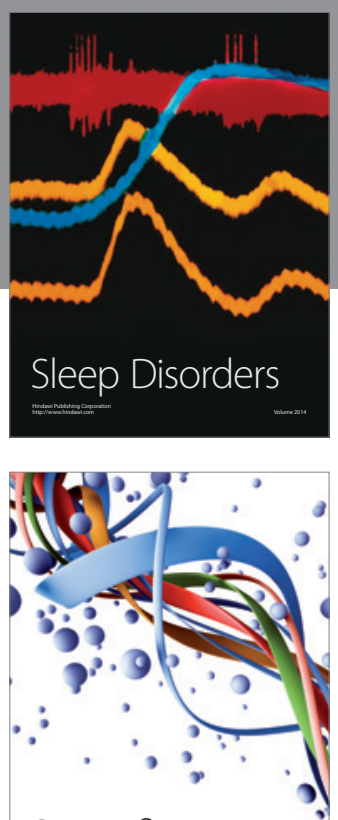

Scientifica
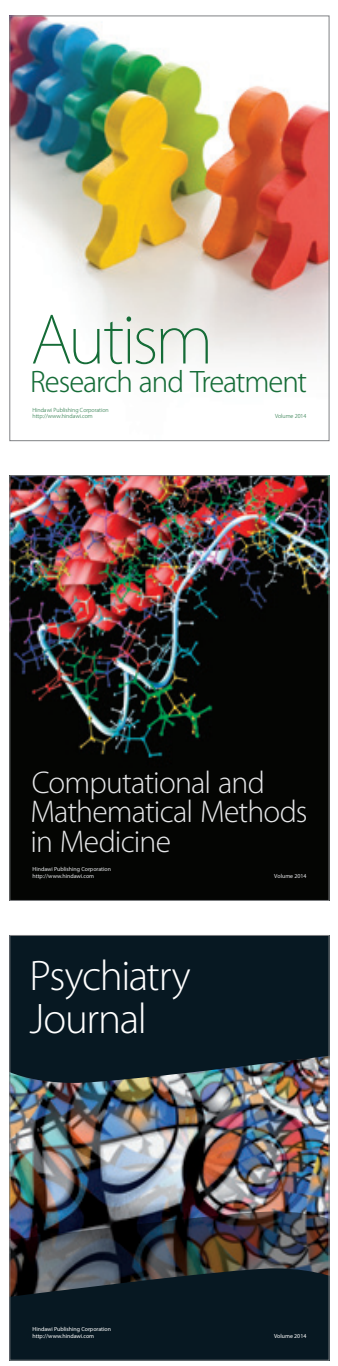
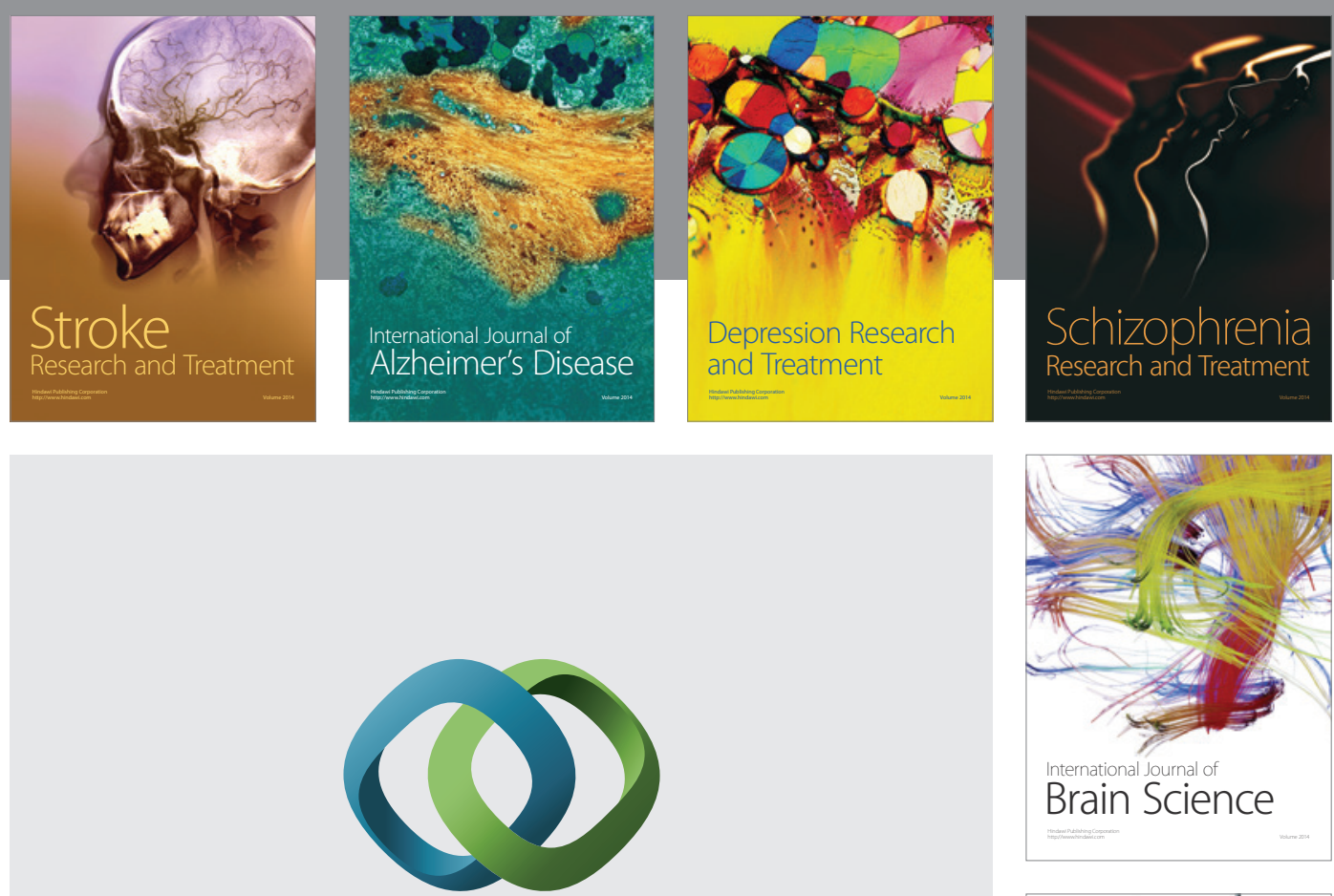

\section{Hindawi}

Submit your manuscripts at

http://www.hindawi.com
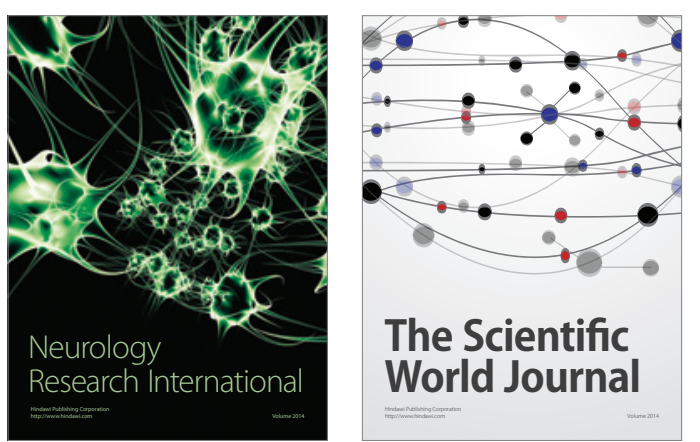

The Scientific World Journal

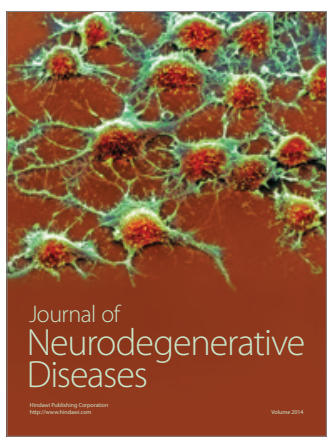

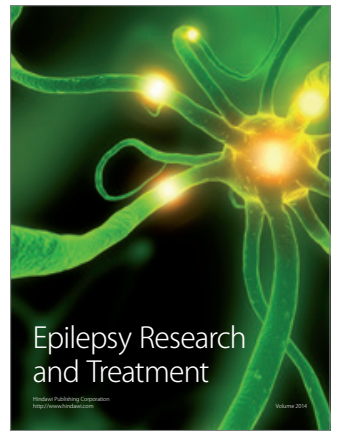

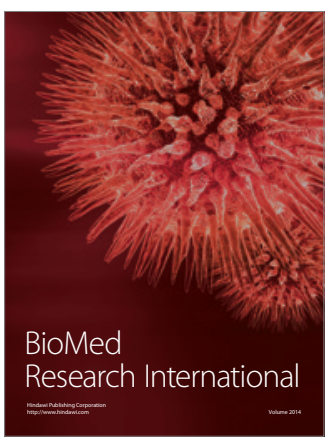

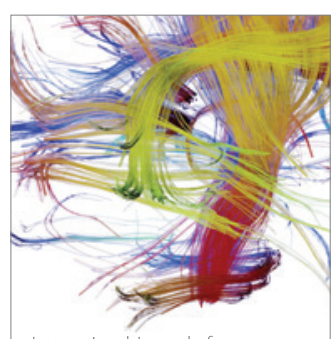

Brain Science

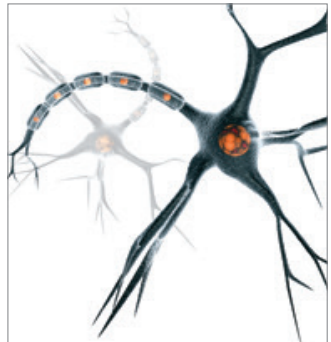

Neural Plasticity
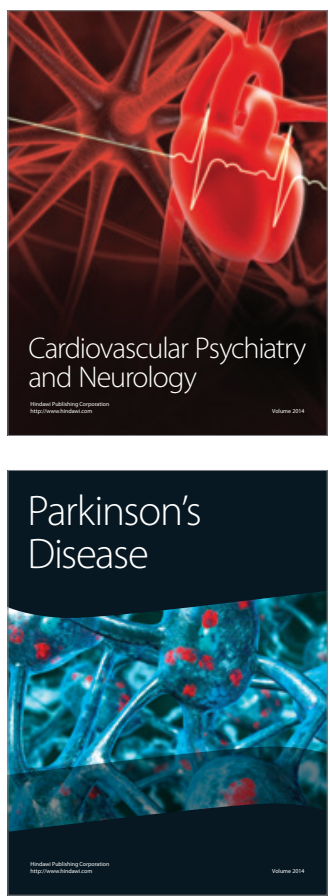